\title{
Distributions and Variations of Two Estuarine Species of Composetia (Annelida: Nereididae) in the Ryukyu Islands, Southern Japan, with a New Record of Composetia tokashikiensis from Thailand
}

\author{
Masanori Sato ${ }^{1,6}$, Takumi Ebihara ${ }^{1}$, Kiyoshi Satake², Shigeaki Kojima ${ }^{3}$, \\ Hiroaki Fukumori ${ }^{3,4}$, and Saowapa Angsupanich ${ }^{5}$ \\ ${ }^{1}$ Graduate School of Science and Engineering, Kagoshima University, Kagoshima 890-0065, Japan \\ E-mail: sato@sci.kagoshima-u.ac.jp \\ ${ }_{2}^{2}$ National Institute for Environmental Studies, Tsukuba, Ibaraki 305-8506, Japan \\ ${ }^{3}$ Atmosphere and Ocean Research Institute, The University of Tokyo, Kashiwanoha, Kashiwa, Chiba 277-8564, Japan \\ ${ }^{4}$ Sesoko Station, Tropical Biosphere Research Center, University of the Ryukyus, Sesoko, Motobu, Okinawa 905-0227, Japan \\ ${ }^{5}$ Marine and Coastal Resources Institute, Prince of Songkla University, Hat Yai, Songkhla 90110, Thailand \\ ${ }^{6}$ Corresponding author
}

(Received 2 May 2018; Accepted 18 November 2019)

\begin{abstract}
By the survey on the nereidid fauna in small estuaries in creeks in the Ryukyu Islands, southern Japan, two species of Composetia Hartmann-Schröder, 1985, C. kumensis Sato, 2020 (type locality: Kume-jima island, the Ryukyu Islands) and C. tokashikiensis Sato, 2020 (type locality: Tokashiki-jima island, the Ryukyu Islands) have been newly collected from 10 sites on 5 islands, and from 20 sites on 6 islands, respectively. In 26 of a total of 28 sites, one of the two species occurred exclusively, but both of them coexisted in two sites in Oura Bay on Okinawa-jima island. Morphological variations and genetic differentiations among the local populations of the two species were examined. Molecular analyses based on the partial sequences of the mitochondrial cytochrome $c$ oxidase subunit I gene supported that the morphologically similar two species are certainly distinct species. In C. kumensis, marked genetic differentiation was detected among populations in the following three regions in the middle Ryukyu Islands: (1) northern Kume-jima, (2) southern Kume-jima, and (3) Okinawa-jima and Kikai-jima islands. In contrast, no marked genetic differentiation was detected among any populations of C. tokashikiensis in a wide range of the middle and southern Ryukyu Islands. A single specimen of C. tokashikiensis was also collected from an euryhaline area of the lower reaches of Songkhla Lagoon, southern Thailand. A morphological description of this specimen is provided here as a new record of this species from Thailand.
\end{abstract}

Key Words: Allometry, COI, Composetia kumensis, haplotype, genetic differentiation, paragnath.

\section{Introduction}

Estuaries are an ecologically important habitat as the critical transition zone between freshwater and marine habitats (Levin et al. 2001). In general, some euryhaline species of nereidid polychaetes are major components of the macrobenthic fauna in estuaries, playing important roles in nutrient cycling in an estuary ecosystem (Sato 2017). However, surveys on the nereidid fauna in Asian tropical and subtropical estuaries are insufficient.

Recently, two nereidid species of Composetia HartmannSchröder, 1985, i.e., C. kumensis Sato, 2020 and C. tokashikiensis Sato, 2020, were described from subtropical small estuaries in Kume-jima and Tokashiki-jima islands in the Ryukyu Islands, southern Japan, respectively (Sato 2020). In our extensive surveys to clarify the geographical distributions of these two species, we found their several additional habitats in the middle and southern Ryukyu Islands and along the coast of Thailand. In the present paper, their distributions, interpopulation morphological variations, and genetic divergence are reported.

\section{Materials and Methods}

Collection of specimens and identification to species. Specimens of Composetia were collected from 28 small estuaries of nine islands in the middle and southern Ryukyu Islands, southern Japan from 1999 to 2017, and also from the lower reaches of Songkhla Lagoon, southern Thailand (Fig. 1; Table 1). They were collected from sediment samples dug out from intertidal or shallow subtidal bottoms using shovels or Tamura grab, and fixed in $10 \%$ formalin or $80 \%$ ethanol and transferred to 80 or $99 \%$ ethanol for preservation. Water temperature and salinity in the sampling sites were measured with a SCT meter (Model 30, Yellow Springs Instrument, Co.). The specimens were identified to species according to Sato (2020).

Morphological observation. For preserved specimens, anterior maximum body width excluding parapodia (BW) was measured, and anterior chaetigers with the neuropodial postchaetal lobe were counted; for complete specimens only, body length from the anterior end of the prostomium to the posterior end of the pygidium excluding the anal 


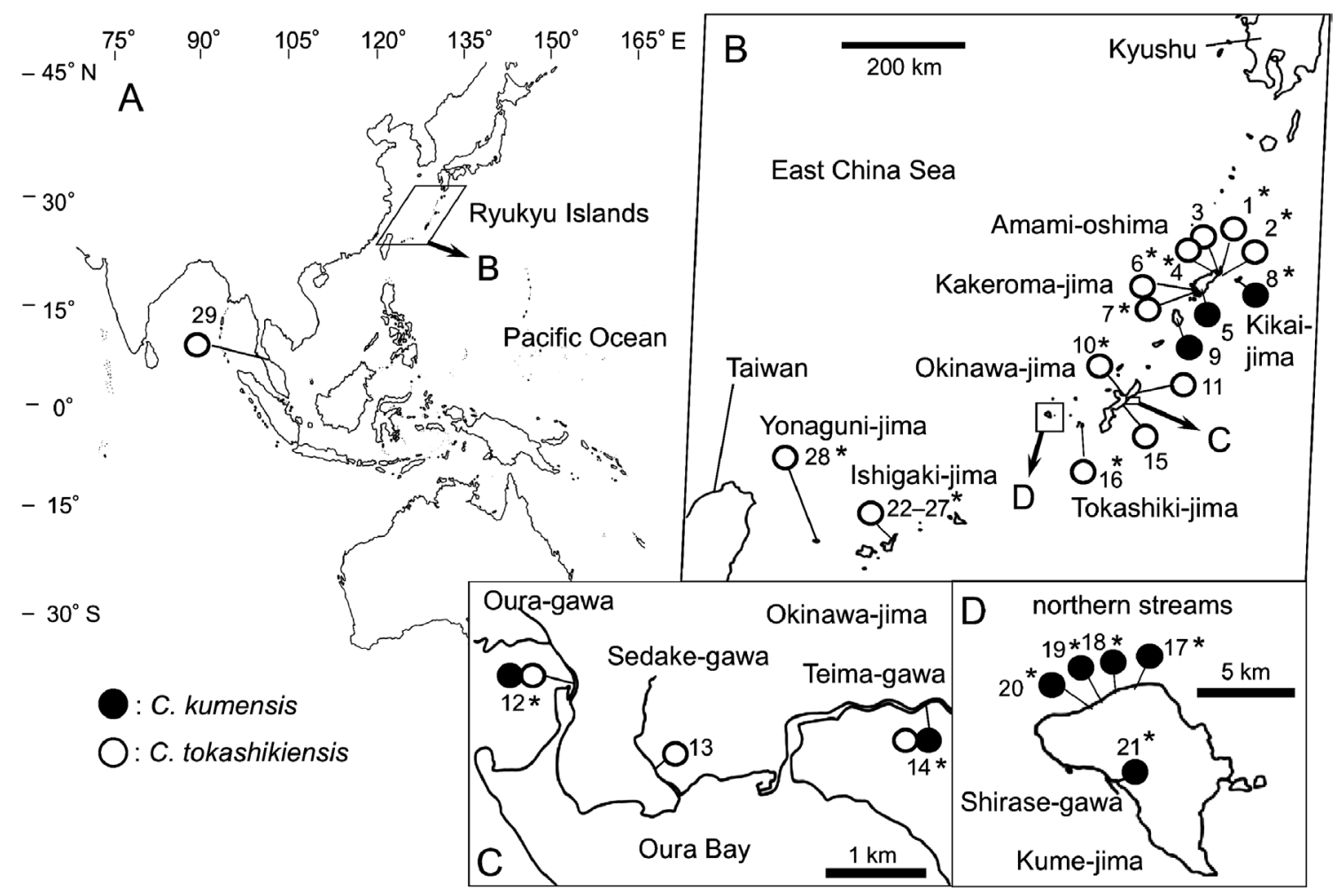

Fig. 1. Distributions of two species of Composetia. *Mitochondrial COI gene was analyzed.

cirri (BL) was measured, and number of total chaetigers was also counted. The paragnaths in each area on the proboscis were counted under an Olympus SZH10 stereoscopic microscope; numbers of paragnaths in the areas II and IV were represented by the larger number in either right or left sides. When coelomic oocytes were found in the preserved specimens, the longest diameter of 5-10 randomly chosen oocytes was measured with a micrometer in a Nikon Optiphot-2 compound microscope. Data of type series of the two species of Composetia (Sato 2020) were also used together with those of the newly collected specimens in the present study for the comparisons among geographically separated local populations. Photographs of the specimens were taken with a Nikon D3200 digital camera, and another one (Nikon Coolpix) adapted on a Nikon SMZ1000 stereoscopic microscope. Drawings were prepared with a camera lucida.

Terminology of paragnath groups on proboscis, and that of parapodial and chaetal morphology are according to Bakken and Wilson (2005).

Abbreviations of depositories of the specimens are as follows: NSMT, National Museum of Nature and Science, Tsukuba, Japan; PMBC, the Phuket Marine Biological Center, Phuket, Thailand.

Molecular analyses. Total DNA was extracted from each specimen with a DNeasy Tissue Extraction Kit (Qiagen, Valencia, CA, USA) according to the manufacturer's instructions. By using each total DNA as a template, a part (960 bp) of the mitochondrial cytochrome $c$ oxidase subunit I (COI) gene was amplified by polymerase chain reaction (PCR) with primers COI-B (Hasegawa et al. 1996) and
COI-6 (Shimayama et al. 1990). The conditions for PCR were $94^{\circ} \mathrm{C}$ for $60 \mathrm{~s}$ followed by $30-40$ cycles at $94^{\circ} \mathrm{C}$ for $40 \mathrm{~s}$, $50^{\circ} \mathrm{C}$ for $60 \mathrm{~s}$, and $72^{\circ} \mathrm{C}$ for $90 \mathrm{~s}$, and finally an extension at $72^{\circ} \mathrm{C}$ (7 min). GeneReleaser (BioVenture Inc., Murfreesboro, TN, USA) was used to sequester the products of cell lysis that might have inhibited the polymerase. The nucleotide sequences (694 bp) of the amplified fragments were determined using an automated sequencer (ABI3130, Applied Biosystems Inc., CA, USA) with the primers COI-B, COI-3 (Shimayama et al. 1990), TW-2 (Kojima et al. 1997), and/ or COI-6. The nucleotide sequences reported in the present study have been deposited in the DDBJ/EMBL/GenBank databases under accession numbers LC498532-LC498556.

COI haplotype relationships were analyzed using the Bayesian, maximum-likelihood (ML), maximum-parsimony (MP), and neighbor-joining (NJ) methods. The Bayesian tree was reconstructed using MrBayes ver. 3.1.2 (Huelsenbeck and Ronquist 2001; Ronquist and Huelsenbeck 2003) with a General-Time-Reversible (GTR)+Gamma (G)+Proportion Invariant (I) substitution model. The shape, proportion of invariant sites, state frequency and substitution rate parameters were separately estimated for each codon position. Two parallel runs were made for $10^{7}$ generations (with a sample frequency of 1000) using the default value of four Markov chains. The first 1000 trees for each run were discarded to ensure the four chains reached stationarity by referring to the average standard deviation of split frequencies (Ronquist and Huelsenbeck 2003). The consensus tree and posterior probabilities were computed from the remaining 18000 trees (9000 trees $\times 2$ runs). The ML tree was constructed using RAxML 7.4.2 (Stamatakis 
Table 1. Collection data of two species of Composetia.

\begin{tabular}{|c|c|c|c|c|c|}
\hline \multirow{2}{*}{$\begin{array}{c}\text { Site } \\
\text { number }\end{array}$} & \multirow{2}{*}{ Locality of collection sites (Habitat type ${ }^{2}$ ) } & \multicolumn{2}{|c|}{ Date of collection (no. of specimens) ${ }^{3}$} & \multirow{2}{*}{$\begin{array}{l}\text { Associated fauna and } \\
\text { flora }\end{array}$} & \multirow{2}{*}{ Collector } \\
\hline & & C. kumensis & C. tokashikiensis & & \\
\hline \multicolumn{6}{|c|}{ Amami-oshima island } \\
\hline 1 & Yani-gawa river (B); $28^{\circ} 29^{\prime} 52.1^{\prime \prime} \mathrm{N}, 129^{\circ} 39^{\prime} 26.22^{\prime \prime} \mathrm{E}$ & & 17 May $2014(4)^{*}$ & & M. Sato et al. \\
\hline 2 & Mae-kawa river (B); $28^{\circ} 27^{\prime} 2.07^{\prime \prime} \mathrm{N}, 129^{\circ} 42^{\prime} 52.04^{\prime \prime} \mathrm{E}$ & & 17 May $2014(6)^{*}$ & & M. Sato et al. \\
\hline 3 & Kise, Miyakuda-gawa river (B); $28^{\circ} 25^{\prime} 17.43^{\prime \prime} \mathrm{N}, 129^{\circ} 39^{\prime} 6.06^{\prime \prime} \mathrm{E}$ & & 21 August 2010 (1) & Hediste atoka & T. Ebihara \\
\hline 4 & Ura, Tatsugo-cho (B); $28^{\circ} 24^{\prime} 47.33^{\prime \prime} \mathrm{N}, 129^{\circ} 35^{\prime} 25.9^{\prime \prime} \mathrm{E}$ & & $\begin{array}{l}3 \text { November } 2008(1)^{*} \text {, } \\
21 \text { August } 2010(29)\end{array}$ & Hediste atoka, Mangroves & T. Ebihara \\
\hline 5 & Atetsu, Setouchi-cho (B); $28^{\circ} 11^{\prime} 26.1^{\prime \prime} \mathrm{N}, 129^{\circ} 17^{\prime} 30.6^{\prime \prime} \mathrm{E}$ & 12 January $2016(7)$ & & & N. Jimi \\
\hline \multicolumn{6}{|c|}{ Kakeroma-jima island } \\
\hline 6 & Nakata-gawa river $(\mathrm{B}) ; 28^{\circ} 8^{\prime} 42.15^{\prime \prime} \mathrm{N}, 129^{\circ} 12^{\prime} 45.22^{\prime \prime} \mathrm{E}$ & & 18 May $2014(2)^{*}$ & & K. Kan \\
\hline 7 & Yamada-gawa river (B); $28^{\circ} 6^{\prime} 40.45^{\prime \prime} \mathrm{N}, 129^{\circ} 13^{\prime} 21.37^{\prime \prime} \mathrm{E}$ & & 18 May $2014(10)^{*}$ & Ptychognathus sp. & K. Kan \\
\hline \multicolumn{6}{|c|}{ Kikai-jima island } \\
\hline 8 & Urabaru-gawa river $(\mathrm{A}) ; 28^{\circ} 17^{\prime} 1.54^{\prime \prime} \mathrm{N}, 129^{\circ} 57^{\prime} 49.93^{\prime \prime} \mathrm{E}$ & $2008-2009(114)^{*}$ & & $\begin{array}{c}\text { Hediste atoka, } \\
\text { oligochaetes }\end{array}$ & T. Ebihara \\
\hline \multicolumn{6}{|c|}{ Tokuno-shima island } \\
\hline \multirow[t]{2}{*}{9} & Senma-kaigann, Amagi-cho (A); $27^{\circ} 47^{\prime} 6.27^{\prime \prime} \mathrm{N}, 128^{\circ} 53^{\prime} 38.75^{\prime \prime} \mathrm{E}$ & 18 June 2016 (1) & & & K. Kan \\
\hline & & 12 April 2017 (7) & & & M. Sato et al. \\
\hline \multicolumn{6}{|c|}{ Okinawa-jima island } \\
\hline 10 & Gabui, Minato-gawa river (B); $26^{\circ} 39^{\prime} 4.3^{\prime \prime} \mathrm{N}, 127^{\circ} 58^{\prime} 54.68^{\prime \prime} \mathrm{E}$ & & 28 June $2008(10)^{*}$ & & T. Ebihara \\
\hline 11 & Higashi-son, Shii-kawa river (B); $26^{\circ} 37^{\prime} 39.16^{\prime \prime} \mathrm{N}, 128^{\circ} 8^{\prime} 47.48^{\prime \prime} \mathrm{E}$ & & 30 June 2008 (5) & & T. Ebihara \\
\hline \multirow[t]{2}{*}{12} & Oura-gawa river, Oura Bay (B); $26^{\circ} 33^{\prime} 36.4^{\prime \prime} \mathrm{N}, 128^{\circ} 02^{\prime} 36.77^{\prime \prime} \mathrm{E}$ & 11 November $2014(6)^{*}$ & 11 November $2014(1)^{*}$ & $\begin{array}{l}\text { Ptychognathus sp., } \\
\text { oligochaetes }\end{array}$ & M. Sato \\
\hline & & 17 December 2014 (2) & & oligochaetes & T. Sakaguchi \\
\hline 13 & Sedake-gawa river, Oura Bay $(\mathrm{B}) ; 26^{\circ} 33^{\prime} 08^{\prime \prime} \mathrm{N}, 128^{\circ} 03^{\prime} 04^{\prime \prime} \mathrm{E}$ & & 17 December 2014 (5) & & T. Sakaguchi \\
\hline \multirow[t]{2}{*}{14} & Teima-gawa river, Oura Bay (B); $26^{\circ} 33^{\prime} 25.89^{\prime \prime} \mathrm{N}, 128^{\circ} 04^{\prime} 41.22^{\prime \prime} \mathrm{E}$ & 11 November $2014(4)^{*}$ & 11 November $2014(7) *$ & Ptychognathus sp. & M. Sato \\
\hline & & 17 December $2014(2)$ & 17 December 2014 (17) & Tmethypocoelis choreutes & T. Sakaguchi \\
\hline 15 & Okukubi-gawa river, Kin-cho (B); $26^{\circ} 27^{\prime} 45.35^{\prime \prime} \mathrm{N}, 127^{\circ} 55^{\prime} 58.95^{\prime \prime} \mathrm{E}$ & & 10 September $2004(17)$ & & \\
\hline \multicolumn{6}{|c|}{ Tokashiki-jima island } \\
\hline \multirow[t]{2}{*}{16} & Tokashiki-gawa river (B); $26^{\circ} 11^{\prime} 46.81^{\prime \prime} \mathrm{N}, 127^{\circ} 21^{\prime} 46.82^{\prime \prime} \mathrm{E}$ & & 20 November 1991 (12) & oligochaetes & M. Sato \\
\hline & & & 27 March $2012(9)^{*}$ & Tmethypocoelis choreutes & M. Sato \\
\hline \multicolumn{6}{|c|}{ Kume-jima island } \\
\hline 17 & Hiyajou (A); $26^{\circ} 23^{\prime} 04.84^{\prime \prime} \mathrm{N}, 126^{\circ} 47^{\prime} 10.42^{\prime \prime} \mathrm{E}$ & 26 November $2013(8)^{*}$ & & oligochaetes & M. Sato et al. \\
\hline 18 & Tachijami (A); $26^{\circ} 23^{\prime} 07.7^{\prime \prime} \mathrm{N}, 126^{\circ} 46^{\prime} 02.6^{\prime \prime} \mathrm{E}$ & 24 November $2013(4)^{*}$ & & Ptychognathus sp. & M. Sato et al. \\
\hline 19 & East of Mifuga (A); $26^{\circ} 22^{\prime} 58.7^{\prime \prime} \mathrm{N}, 126^{\circ} 45^{\prime} 32.8^{\prime \prime} \mathrm{E}$ & 22 November $2013(4)^{*}$ & & & M. Sato et al. \\
\hline \multirow[t]{2}{*}{20} & Gushicha Gusuku (A); $26^{\circ} 22^{\prime} 52.6^{\prime \prime} \mathrm{N}, 126^{\circ} 45^{\prime} 15.2^{\prime \prime} \mathrm{E}$ & $\begin{array}{l}25 \text { March } 1999 \text { (3), } \\
23 \text { March } 2007 \text { (2) }\end{array}$ & & & K. Satake \\
\hline & & 22 November $2013(14)^{*}$ & & Ptychognathus spp. & M. Sato et al. \\
\hline 21 & Shirase-gawa river (B); $26^{\circ} 20^{\prime} 38.4^{\prime \prime} \mathrm{N}, 126^{\circ} 45^{\prime} 49.0^{\prime \prime} \mathrm{E}$ & 24 January $2014(3)^{*}$ & & & K. Satake \\
\hline \multicolumn{6}{|c|}{ Ishigaki-jima island } \\
\hline 22 & Creek in Hirakubo (B); $24^{\circ} 35^{\prime} 16.46^{\prime \prime} \mathrm{N}, 124^{\circ} 18^{\prime} 44.70^{\prime \prime} \mathrm{E}$ & & 9 March $2015(4)^{*}$ & & K. Kan \\
\hline 23 & Kara-gawa river (B); $24^{\circ} 34^{\prime} 7.61^{\prime \prime} \mathrm{N}, 124^{\circ} 17^{\prime} 51.85^{\prime \prime} \mathrm{E}$ & & 11 September 2009 (7) & & T. Ebihara \\
\hline 24 & Ohura-gawa river, Iharama (B); $24^{\circ} 30^{\prime} 32^{\prime \prime} \mathrm{N}, 124^{\circ} 16^{\prime} 12.5^{\prime \prime} \mathrm{E}$ & & 8 March $2015(5) *$ & & K. Kan \\
\hline 25 & Inoda-bridge, Inoda-gawa river (B); $24^{\circ} 27^{\prime} 53.38^{\prime \prime} \mathrm{N}, 124^{\circ} 15^{\prime} 0.14^{\prime \prime} \mathrm{E}$ & & 8 March $2015(16)^{*}$ & & K. Kan \\
\hline 26 & Bridge, Touro-gawa river (B); $24^{\circ} 25^{\prime} 58.2^{\prime \prime} \mathrm{N}, 124^{\circ} 14^{\prime} 53.3^{\prime \prime} \mathrm{E}$ & & 8 March $2015(9)^{*}$ & & K. Kan \\
\hline 27 & Anparu, Haikah-bridge (B); $24^{\circ} 23^{\prime} 27.7^{\prime \prime} \mathrm{N}, 124^{\circ} 9^{\prime} 2.6^{\prime \prime} \mathrm{E}$ & & 7 March $2015(2)^{*}$ & & K. Kan \\
\hline \multicolumn{6}{|c|}{ Yonaguni-jima island } \\
\hline 28 & Tabaru-gawa river $(\mathrm{B}) ; 24^{\circ} 27^{\prime} 58.00^{\prime \prime} \mathrm{N}, 123^{\circ} 0^{\prime} 8.23^{\prime \prime} \mathrm{E}$ & & 4 March $2015(12)^{*}$ & Mangroves & K. Kan \\
\hline \multicolumn{6}{|l|}{ Thailand } \\
\hline 29 & Songkhla Lagoon; $7^{\circ} 10^{\prime} 37.4^{\prime \prime} \mathrm{N}, 100^{\circ} 32^{\prime} 26.2^{\prime \prime} \mathrm{E}$ & & 19 November 2008 (1) & & $\begin{array}{l}\text { S. Angsupa- } \\
\text { nich }\end{array}$ \\
\hline
\end{tabular}

${ }^{1}$ Corresponding to site numbers in Fig. $1 .{ }^{2} \mathrm{~A}$ and B corresponding to the stream and river types, respectively, shown in Figs $2,3 .{ }^{3}$ Asterisks mean that mitochondrial COI gene was analyzed.

2006), which followed a GTR $+\mathrm{G}$ substitution model. For the RAxML analysis, the data were partitioned by codon position and bootstrapped with 1000 replicates following the software manual. The MP tree was constructed using MEGA 5 (Tamura et al. 2011) with the Subtree-Pruning-Regrafting (SPR) option with 1000 replicates. The NJ tree was constructed using MEGA 5 with Kimura's two-parameter method (Kimura 1980) and bootstrapped with 1000 repli- cates. In all analyses, the nucleotide sequences of a specimen of the confamiliar species "Neanthes japonica (Izuka, 1908)" (Kojima et al. 1997; DDBJ/EMBL/GenBank accession number: D38032), which was judged to belong to Hediste atoka Sato and Nakashima, 2003 based on a taxonomic revision of Hediste Malmgren, 1867 from Japan (Sato and Nakashima 2003), was used as an outgroup. 


\section{Results}

Distribution and habitat of the two species of Composetia in the Ryukyu Islands. A total of 181 specimens of C. kumensis collected from 10 sites on five islands in the middle Ryukyu Islands (from the north to Kikai-jima island to the south to Kume-jima island) were used in the present study, with 162 specimens newly collected from 9 sites added to 19 specimens of the type series of this species (Sato 2020) (Tables 1, 2). A total of 191 specimens of C. tokashikiensis collected from 20 sites on six islands in the middle and southern Ryukyu Islands (from the north to Amami-oshima island to the south to Yonaguni-jima island) were used in the present study, with 170 specimens newly collected from 19 sites added to 21 specimens of the type series of this species (Sato 2020) (Tables 1, 3). In most habitats (26 sites), either species occurred exclusively, but both of the species coexisted in the neighboring two rivers, Oura-gawa $(5 \mathrm{~km}$ in riverine length; Maeda and Tachihara 2006) and Teimagawa $(6.7 \mathrm{~km})$ rivers, which flow into the Oura Bay on the east coast of Okinawa-jima island, with a distance of $2.3 \mathrm{~km}$ between their mouths (Fig. 1).

All specimens of the two species were collected from around the uppermost reaches of small estuaries (around the upper limit of the rising seawater in spring tides, between the high tide marks of spring tides and neap tides). Their habitats could be classified into the following two types: (1) the stream type, with a small amount of stable freshwater flow (usually less than $1 \mathrm{~m}$ wide), located on the upper intertidal zone of the uplifted coral reefs, and originated from a freshwater spring inside or outside of the intertidal zone (Figs 2A, 3A); (2) the usual river type, with a relatively large amount of freshwater flow (usually more than $1 \mathrm{~m}$ wide) (Figs 2B, 3B). Composetia tokashikiensis was found only from the river type, whereas C. kumensis was found from both of the stream and river types (Table 1).

Their habitats seem to be usually under a freshwater regime, but sometimes exposed to nearly full-strength seawater for a few hours around high-tide time during the extreme spring tides, when their habitats are submerged (Sato 2020). In fact, the salinity of the bottom water in the habitat of C. kumensis in Urabaru-gawa river on Kikai-jima island varied from 0 at low tide to 34 at high tide in September 2009 (our unpublished data).

Associated fauna of macrobenthic organisms was generally very poor in the habitats of both species of Composetia, though the nereidid polychaete Hediste atoka, oligochaetes, the dotillid crab Tmethypocoelis choreutes Davie and Kosuge, 1995, and varunid crabs of the genus Ptychognathus Stimpson, 1858 were found coexisting with the species of Composetia in some habitats (Table 1). Mangrove trees of Rhizophoraceae were also found in two sites of habitats of the species of Composetia.

Genetic differentiations of the two species of Composetia in the Ryukyu Islands. We examined the genetic differentiation among 23 local populations of the two species (8 localities for C. kumensis, and 15 localities for C. tokashi-

Table 2. Variation of morphological characteristics in 10 local populations of Composetia kumensis.

\begin{tabular}{|c|c|c|c|c|c|c|c|c|c|c|}
\hline \multirow{2}{*}{ Locality (site no.) ${ }^{1}$} & \multirow{2}{*}{$\begin{array}{c}\mathrm{n} \\
\text { (catalogue } \\
\text { no. })^{2}\end{array}$} & \multirow{2}{*}{$\begin{array}{l}\text { Body } \\
\text { width } \\
(\mathrm{mm})\end{array}$} & \multirow{2}{*}{$\begin{array}{l}\text { Body } \\
\text { length } \\
(\mathrm{mm})\end{array}$} & \multirow{2}{*}{$\begin{array}{c}\text { No. of } \\
\text { total } \\
\text { chaetigers }^{3}\end{array}$} & \multirow{2}{*}{$\begin{array}{l}\text { No. of anterior } \\
\text { chaetigers }^{4}\end{array}$} & \multicolumn{5}{|c|}{ No. of paragnaths ${ }^{5}$} \\
\hline & & & & & & I & $\mathrm{II}^{6}$ & III & $\mathrm{IV}^{6}$ & Total \\
\hline \multicolumn{11}{|l|}{ Amami-oshima } \\
\hline Atetsu (5) & $7(113255)$ & $0.8-1.2$ & $12-17(3)$ & $55-58(3)$ & $\begin{array}{l}8.4 \pm 0.5 \\
(8-9,7)\end{array}$ & $\begin{array}{l}0.2 \pm 0.4 \\
(0-1,6)\end{array}$ & $\begin{array}{l}22.3 \pm 3.9 \\
(16-26,6)\end{array}$ & $\begin{array}{l}15.8 \pm 6.1 \\
(8-25,6)\end{array}$ & $\begin{array}{l}23.8 \pm 2.9 \\
(21-27,6)\end{array}$ & $\begin{array}{l}105.5 \pm 16.3 \\
(84-120,6)\end{array}$ \\
\hline \multicolumn{11}{|l|}{ Kikai-jima } \\
\hline Urabaru-gawa (8) & $\begin{array}{c}114 \\
(113256)\end{array}$ & $0.6-1.4$ & $6-20(96)$ & $32-63(96)$ & $\begin{array}{c}9.6 \pm 1.6 \\
(6-14,100)\end{array}$ & $\begin{array}{c}0 \pm 0 \\
(0-0,114)\end{array}$ & $\begin{array}{c}23.1 \pm 3.1 \\
(16-36,112)\end{array}$ & $\begin{array}{c}12.1 \pm 2.2 \\
(7-18,112)\end{array}$ & $\begin{array}{c}17.0 \pm 2.5 \\
(11-23,112)\end{array}$ & $\begin{array}{c}87.7 \pm 9.9 \\
(64-114,112)\end{array}$ \\
\hline \multicolumn{11}{|l|}{ Tokuno-shima } \\
\hline Senma-kaigann (9) & $\begin{array}{c}8(113257- \\
113259)\end{array}$ & $0.8-1.2$ & $18(1)$ & $56(1)$ & $\begin{array}{l}7.4 \pm 1.2 \\
(6-9,8)\end{array}$ & $\begin{array}{c}0 \pm 0 \\
(0-0,8)\end{array}$ & $\begin{array}{l}24.5 \pm 3.2 \\
(19-29,8)\end{array}$ & $\begin{array}{c}17.0 \pm 2.3 \\
(14-21,8)\end{array}$ & $\begin{array}{c}19.6 \pm 2.0 \\
(17-22,8)\end{array}$ & $\begin{array}{l}100.1 \pm 9.3 \\
(87-112,8)\end{array}$ \\
\hline \multicolumn{11}{|l|}{ Okinawa-jima } \\
\hline Oura-gawa (12) & $\begin{array}{c}8(113260, \\
113261)\end{array}$ & $0.6-1.0$ & $8-13(3)$ & $44-51(3)$ & $\begin{array}{l}5.1 \pm 1.3 \\
(4-8,7)\end{array}$ & $\begin{array}{c}0 \pm 0 \\
(0-0,6)\end{array}$ & $\begin{array}{l}27.8 \pm 2.6 \\
(24-31,6)\end{array}$ & $\begin{array}{c}16.5 \pm 2.5 \\
(13-19,8)\end{array}$ & $\begin{array}{l}21.4 \pm 2.4 \\
(18-24,5)\end{array}$ & $\begin{array}{l}111.6 \pm 8.5 \\
(99-119,5)\end{array}$ \\
\hline Teima-gawa (14) & $\begin{array}{c}6(113262 \\
113263)\end{array}$ & $0.6-0.9$ & $8-9(4)$ & $39-49(2)$ & $\begin{array}{l}6.0 \pm 0.6 \\
(5-7,6)\end{array}$ & $\begin{array}{c}0 \pm 0 \\
(0-0,4)\end{array}$ & $\begin{array}{l}27.5 \pm 2.6 \\
(24-30,4)\end{array}$ & $\begin{array}{c}15.4 \pm 1.5 \\
(14-17,5)\end{array}$ & $\begin{array}{l}22.8 \pm 1.7 \\
(21-25,4)\end{array}$ & $\begin{array}{c}110.5 \pm 7.2 \\
(101-117,4)\end{array}$ \\
\hline \multicolumn{11}{|l|}{ Kume-jima } \\
\hline Hiyajou (17) & $8(113264)$ & $0.7-1.3$ & $9-24(3)$ & $43-56(3)$ & $\begin{array}{l}10.8 \pm 1.3 \\
(9-13,6)\end{array}$ & $\begin{array}{l}0.4 \pm 0.9 \\
(0-2,5)\end{array}$ & $\begin{array}{l}22.0 \pm 1.6 \\
(20-24,5)\end{array}$ & $\begin{array}{l}9.6 \pm 2.4 \\
(7-12,5)\end{array}$ & $\begin{array}{c}18.2 \pm 2.8 \\
(15-22,5)\end{array}$ & $\begin{array}{l}86.8 \pm 6.2 \\
(81-96,5)\end{array}$ \\
\hline Tachijami (18) & $4(113265)$ & $1.0-1.2$ & $12-16(4)$ & $54-55(4)$ & $\begin{array}{l}8.3 \pm 0.5 \\
(8-9,4)\end{array}$ & $\begin{array}{l}0.5 \pm 0.6 \\
(0-1,4)\end{array}$ & $\begin{array}{l}18.5 \pm 1.3 \\
(17-20,4)\end{array}$ & $\begin{array}{l}11.8 \pm 3.9 \\
(8-17,4)\end{array}$ & $\begin{array}{c}15.0 \pm 2.2 \\
(13-18,4)\end{array}$ & $\begin{array}{l}76.5 \pm 11.6 \\
(66-93,4)\end{array}$ \\
\hline Mifuga (19) & $4(113266)$ & $0.8-1.2$ & $12-13(3)$ & $53-55(3)$ & $\begin{array}{l}7.3 \pm 1.0 \\
(6-8,4)\end{array}$ & $\begin{array}{l}0.3 \pm 0.5 \\
(0-1,4)\end{array}$ & $\begin{array}{l}22.0 \pm 1.7 \\
(21-24,3)\end{array}$ & $\begin{array}{c}12.8 \pm 1.7 \\
(11-15,4)\end{array}$ & $\begin{array}{c}18.3 \pm 2.9 \\
(15-20,3)\end{array}$ & $\begin{array}{l}89.3 \pm 10.6 \\
(78-99,3)\end{array}$ \\
\hline Gushicha Gusuku $(20)^{7}$ & $\begin{array}{l}19(\mathrm{H}-766, \\
\text { P-767-773) }\end{array}$ & $0.7-1.5$ & $9-17(13)$ & $49-64(13)$ & $\begin{array}{c}8.3 \pm 1.2 \\
(6-10,19)\end{array}$ & $\begin{array}{c}0.6 \pm 0.7 \\
(0-2,18)\end{array}$ & $\begin{array}{c}24.7 \pm 3.4 \\
(20-32,17)\end{array}$ & $\begin{array}{c}17.0 \pm 2.9 \\
(13-25,17)\end{array}$ & $\begin{array}{c}22.4 \pm 3.3 \\
(19-28,17)\end{array}$ & $\begin{array}{c}105.4 \pm 12.3 \\
(89-134,16)\end{array}$ \\
\hline Shirase-gawa (21) & $3(113268)$ & $0.7-1.2$ & $12-16(2)$ & $47-52(2)$ & $\begin{array}{l}7.3 \pm 1.2 \\
(6-8,3)\end{array}$ & $\begin{array}{c}1.5 \\
(1-2,2)\end{array}$ & $\begin{array}{c}20.0 \\
(18-22,2)\end{array}$ & $\begin{array}{c}11.5 \\
(9-14,2)\end{array}$ & $\begin{array}{c}16.5 \\
(16-17,2)\end{array}$ & $\begin{array}{c}81.0 \\
(77-85,2)\end{array}$ \\
\hline
\end{tabular}

${ }^{1}$ Corresponding to site numbers in Fig. 1 and Table $1 .{ }^{2}$ Catalogue numbers for the specimen depository in NSMT-Pol. ${ }^{3}$ Data of only complete specimens. Range $(n)$.

${ }^{4}$ Chaetigrs with neuropodial postchaetal lobe. Mean $\pm \operatorname{SD}$ (range, $\left.n\right) .{ }^{5}$ Mean $\pm \mathrm{SD}$ (range, $n$ ). ${ }^{6}$ Larger value at a left or right side. ${ }^{7}$ Type locality. 
kiensis; Fig. 1; Table 1) in the Ryukyu Islands by the comparison of the partial sequences of the mitochondrial COI gene. From a total of 73 specimens analyzed, 25 kinds of nucleotide sequences (haplotypes) were obtained (Table 4). All molecular analyses well-supported that C. kumensis and C. tokashikiensis are certainly distinct species since none of the haplotypes was shared by the two species even in their sympatric habitats (Fig. 4).

In C. tokashikiensis, the genetic divergence was very small among all of 14 haplotypes, which were obtained from 15 localities on six islands; the haplotype $\mathrm{C}$ was wide- spread throughout the middle and southern Ryukyu Islands, whereas the other six $(T, U, V, W, X, Y)$ and seven $(A, B, D$, E, F, G, H) haplotypes were restricted to the southern and middle Ryukyu Islands, respectively (Fig. 4; Table 4).

In contrast, a marked genetic divergence was detected among haplotypes of $C$. kumensis in the following three areas: (1) northern Kume-jima island, where all of 12 individuals from 4 neighboring streams belonged to a single haplotype (I), (2) southern Kume-jima island, where 2 individuals from a single river belonged to two haplotypes ( $\mathrm{J}$, $\mathrm{K})$, and (3) Oura Bay in the eastern coast of Okinawa-jima

Table 3. Variation of morphological characteristics in 21 local populations of Composetia tokashikiensis.

\begin{tabular}{|c|c|c|c|c|c|c|c|c|c|c|}
\hline \multirow{2}{*}{ Locality (site no.) ${ }^{1}$} & \multirow{2}{*}{$\underset{(\text { catalogue no. })^{2}}{\mathrm{n}}$} & \multirow{2}{*}{$\begin{array}{l}\text { Body } \\
\text { width } \\
(\mathrm{mm})\end{array}$} & \multirow{2}{*}{$\begin{array}{l}\text { Body } \\
\text { length }{ }^{3} \\
(\mathrm{~mm})\end{array}$} & \multirow{2}{*}{$\begin{array}{c}\text { No. of } \\
\text { total } \\
\text { chaetigers }^{3}\end{array}$} & \multirow{2}{*}{$\begin{array}{c}\text { No. of } \\
\text { anterior } \\
\text { chaetigers }^{4}\end{array}$} & \multicolumn{5}{|c|}{ No. of paragnaths 5} \\
\hline & & & & & & I & $\mathrm{II}^{6}$ & III & $\mathrm{IV}^{6}$ & Total \\
\hline \multicolumn{11}{|l|}{ Amami-oshima } \\
\hline Yani-gawa (1) & $4(113269)$ & $0.8-1.1$ & $15(1)$ & $51(1)$ & $\begin{array}{l}13.7 \pm 0.6 \\
(13-14,3)\end{array}$ & $\begin{array}{c}0 \pm 0 \\
(0-0,4)\end{array}$ & $\begin{array}{l}19.8 \pm 3.3 \\
(16-23,4)\end{array}$ & $\begin{array}{c}24.0 \pm 7.6 \\
(16-31,4)\end{array}$ & $\begin{array}{c}16.0 \pm 1.8 \\
(14-18,4)\end{array}$ & $\begin{array}{l}91.3 \pm 18.5 \\
(75-111,4)\end{array}$ \\
\hline Mae-kawa (2) & $6(113270)$ & $1.2-1.6$ & $19-23(3)$ & $62-64(3)$ & $\begin{array}{c}20.5 \pm 7.4 \\
(15-35,6)\end{array}$ & $\begin{array}{c}0 \pm 0 \\
(0-0,6)\end{array}$ & $\begin{array}{l}23.8 \pm 2.5 \\
(20-26,5)\end{array}$ & $\begin{array}{c}24.0 \pm 2.5 \\
(20-27,6)\end{array}$ & $\begin{array}{c}24.4 \pm 2.6 \\
(21-28,5)\end{array}$ & $\begin{array}{c}115.4 \pm 8.0 \\
(108-128,5)\end{array}$ \\
\hline Kise (3) & $1(113271)$ & 0.9 & 11 & 56 & 10 & 0 & 16 & 22 & 18 & 84 \\
\hline Ura (4) & $\begin{array}{c}30(113272, \\
113273)\end{array}$ & $0.7-1.6$ & $\begin{array}{c}10-26 \\
(22)\end{array}$ & $45-60(22)$ & $\begin{array}{c}26.1 \pm 4.5 \\
(16-37,28)\end{array}$ & $\begin{array}{c}0 \pm 0 \\
(0-0,19)\end{array}$ & $\begin{array}{c}26.1 \pm 2.9 \\
(22-33,19)\end{array}$ & $\begin{array}{c}26.6 \pm 2.8 \\
(22-31,19)\end{array}$ & $\begin{array}{c}23.4 \pm 1.8 \\
(20-27,19)\end{array}$ & $\begin{array}{c}121.1 \pm 10.7 \\
(101-139,19)\end{array}$ \\
\hline \multicolumn{11}{|l|}{ Kakeroma-jima } \\
\hline Nakata-gawa (6) & $2(113274)$ & $1.0-1.2$ & & & $\begin{array}{c}17.5 \\
(17-18,2)\end{array}$ & $\begin{array}{c}0 \\
(0-0,2)\end{array}$ & $\begin{array}{c}26.5 \\
(25-28,2)\end{array}$ & $\begin{array}{c}30.0 \\
(28-32,2)\end{array}$ & $\begin{array}{c}28.0 \\
(24-32,2)\end{array}$ & $\begin{array}{c}135.5 \\
(127-144,2)\end{array}$ \\
\hline Yamada-gawa (7) & $10(113275)$ & $1.1-1.8$ & $21-31(7)$ & $62-72(7)$ & $\begin{array}{c}26.1 \pm 7.5 \\
(16-34,9)\end{array}$ & $\begin{array}{c}0 \pm 0 \\
(0-0,9)\end{array}$ & $\begin{array}{c}21.2 \pm 1.3 \\
(19-23,9)\end{array}$ & $\begin{array}{c}23.4 \pm 2.9 \\
(19-28,10)\end{array}$ & $\begin{array}{l}20.9 \pm 2.9 \\
(16-26,9)\end{array}$ & $\begin{array}{c}101.9 \pm 7.0 \\
(94-111,9)\end{array}$ \\
\hline \multicolumn{11}{|l|}{ Okinawa-jima } \\
\hline Gabui (10) & $10(113276)$ & $0.6-1.2$ & $8-20(10)$ & $46-59(10)$ & $\begin{array}{l}11.8 \pm 1.9 \\
(9-15,10)\end{array}$ & $\begin{array}{c}0 \pm 0 \\
(0-0,10)\end{array}$ & $\begin{array}{c}21.2 \pm 2.7 \\
(16-26,10)\end{array}$ & $\begin{array}{c}21.1 \pm 3.4 \\
(14-26,10)\end{array}$ & $\begin{array}{c}20.0 \pm 2.4 \\
(15-23,10)\end{array}$ & $\begin{array}{c}100.0 \pm 12.4 \\
(69-116,10)\end{array}$ \\
\hline Shii-kawa (11) & $5(113277)$ & $0.7-1.0$ & $11-15(4)$ & $39-56(4)$ & $\begin{array}{l}12.8 \pm 1.6 \\
(11-15,5)\end{array}$ & $\begin{array}{c}0 \pm 0 \\
(0-0,5)\end{array}$ & $\begin{array}{c}23.8 \pm 1.9 \\
(21-26,5)\end{array}$ & $\begin{array}{c}22.8 \pm 2.4 \\
(19-25,5)\end{array}$ & $\begin{array}{l}23.8 \pm 3.6 \\
(20-28,5)\end{array}$ & $\begin{array}{l}114.4 \pm 11.7 \\
(99-128,5)\end{array}$ \\
\hline Oura-gawa (12) & $1(113278)$ & 1.0 & & & 12 & 0 & 25 & 25 & 25 & 121 \\
\hline Sedake-gawa (13) & $5(113279)$ & $0.7-1.8$ & $14(1)$ & $59(1)$ & $\begin{array}{l}17.0 \pm 2.7 \\
(13-20,5)\end{array}$ & $\begin{array}{c}0 \pm 0 \\
(0-0,5)\end{array}$ & $\begin{array}{c}22.3 \pm 1.5 \\
(21-24,4)\end{array}$ & $\begin{array}{c}21.4 \pm 4.9 \\
(14-26,5)\end{array}$ & $\begin{array}{l}21.8 \pm 2.1 \\
(20-24,4)\end{array}$ & $\begin{array}{c}109.0 \pm 2.2 \\
(106-111,4)\end{array}$ \\
\hline Teima-gawa (14) & $\begin{array}{c}24(113280, \\
113281)\end{array}$ & $0.6-1.3$ & $7-17(13)$ & $38-62(13)$ & $\begin{array}{l}10.8 \pm 2.1 \\
(6-15,24)\end{array}$ & $\begin{array}{c}0 \pm 0 \\
(0-0,21)\end{array}$ & $\begin{array}{c}23.2 \pm 3.4 \\
(18-31,21)\end{array}$ & $\begin{array}{c}22.7 \pm 5.2 \\
(10-30,24)\end{array}$ & $\begin{array}{c}21.4 \pm 3.7 \\
(15-28,20)\end{array}$ & $\begin{array}{c}106.4 \pm 14.0 \\
(78-131,20)\end{array}$ \\
\hline Okukubi-gawa (15) & $17(113282)$ & $0.7-1.5$ & $11-18(8)$ & $50-62(8)$ & $\begin{array}{c}17.1 \pm 4.6 \\
(12-29,17)\end{array}$ & $\begin{array}{c}0 \pm 0 \\
(0-0,16)\end{array}$ & $\begin{array}{c}22.0 \pm 3.0 \\
(17-28,16)\end{array}$ & $\begin{array}{c}23.9 \pm 1.5 \\
(21-27,16)\end{array}$ & $\begin{array}{c}19.4 \pm 2.8 \\
(16-24,16)\end{array}$ & $\begin{array}{c}102.7 \pm 9.2 \\
(92-121,16)\end{array}$ \\
\hline \multicolumn{11}{|l|}{ Tokashiki-jima } \\
\hline Tokashiki-gawa $(16)^{7}$ & $\begin{array}{l}21(\mathrm{H}-774, \\
\text { P-775-784) }\end{array}$ & $1.0-1.6$ & $\begin{array}{c}13-21 \\
(11)\end{array}$ & $56-63(10)$ & $\begin{array}{c}18.4 \pm 3.1 \\
(12-25,20)\end{array}$ & $\begin{array}{c}0 \pm 0 \\
(0-0,21)\end{array}$ & $\begin{array}{c}23.5 \pm 2.9 \\
(18-29,21)\end{array}$ & $\begin{array}{c}20.2 \pm 2.7 \\
(14-25,21)\end{array}$ & $\begin{array}{c}19.5 \pm 3.4 \\
(10-26,21)\end{array}$ & $\begin{array}{c}101.3 \pm 11.7 \\
(69-116,20)\end{array}$ \\
\hline \multicolumn{11}{|l|}{ Ishigaki-jima } \\
\hline Hirakubo (22) & $4(113283)$ & $0.7-1.5$ & $7-19(3)$ & $42-57(3)$ & $\begin{array}{l}11.8 \pm 1.9 \\
(9-13,4)\end{array}$ & $\begin{array}{c}0 \pm 0 \\
(0-0,4)\end{array}$ & $\begin{array}{l}21.5 \pm 4.7 \\
(15-26,4)\end{array}$ & $\begin{array}{c}22.3 \pm 7.0 \\
(13-30,4)\end{array}$ & $\begin{array}{c}19.8 \pm 3.8 \\
(15-24,4)\end{array}$ & $\begin{array}{l}102.5 \pm 22.0 \\
(71-122,4)\end{array}$ \\
\hline Kara-gawa (23) & $7(113284)$ & $0.6-1.0$ & $11-14(6)$ & $47-57(6)$ & $\begin{array}{l}11.5 \pm 3.2 \\
(7-16,6)\end{array}$ & $\begin{array}{c}0 \pm 0 \\
(0-0,6)\end{array}$ & $\begin{array}{c}23.2 \pm 4.1 \\
(19-29,6)\end{array}$ & $\begin{array}{c}24.3 \pm 3.1 \\
(21-30,6)\end{array}$ & $\begin{array}{c}18.7 \pm 2.9 \\
(16-23,6)\end{array}$ & $\begin{array}{c}103.0 \pm 6.2 \\
(93-111,6)\end{array}$ \\
\hline Ohura-gawa (24) & $5(113285)$ & $0.7-1.0$ & $11-15(4)$ & $52-65(4)$ & $\begin{array}{c}9.4 \pm 1.1 \\
(8-11,5)\end{array}$ & $\begin{array}{c}0 \pm 0 \\
(0-0,4)\end{array}$ & $\begin{array}{c}25.0 \pm 4.4 \\
(20-30,4)\end{array}$ & $\begin{array}{c}21.6 \pm 3.1 \\
(18-26,5)\end{array}$ & $\begin{array}{l}23.0 \pm 5.6 \\
(18-31,4)\end{array}$ & $\begin{array}{l}108.3 \pm 15.3 \\
(91-128,4)\end{array}$ \\
\hline Inoda-gawa (25) & $16(113286)$ & $0.8-1.8$ & $\begin{array}{c}11-31 \\
(11)\end{array}$ & $57-74(6)$ & $\begin{array}{l}11.7 \pm 3.1 \\
(8-17,9)\end{array}$ & $\begin{array}{c}0 \pm 0 \\
(0-0,16)\end{array}$ & $\begin{array}{l}28.9 \pm 2.7 \\
(26-34,9)\end{array}$ & $\begin{array}{c}28.6 \pm 3.9 \\
(22-37,16)\end{array}$ & $\begin{array}{l}30.1 \pm 4.6 \\
(25-38,9)\end{array}$ & $\begin{array}{c}142.1 \pm 15.5 \\
(123-173,9)\end{array}$ \\
\hline Touro-gawa (26) & $9(113287)$ & $0.8-1.3$ & $11-15(7)$ & $45-56(6)$ & $\begin{array}{l}10.2 \pm 1.3 \\
(9-12,9)\end{array}$ & $\begin{array}{c}0 \pm 0 \\
(0-0,9)\end{array}$ & $\begin{array}{c}22.4 \pm 3.3 \\
(18-26,8)\end{array}$ & $\begin{array}{c}23.7 \pm 6.5 \\
(13-32,9)\end{array}$ & $\begin{array}{c}22.1 \pm 6.4 \\
(14-33,8)\end{array}$ & $\begin{array}{l}107.4 \pm 22.5 \\
(75-141,8)\end{array}$ \\
\hline Anparu (27) & $2(113288)$ & $1.3-1.5$ & $22(1)$ & $60(1)$ & $\begin{array}{l}13 \\
(1)\end{array}$ & $\begin{array}{c}0 \\
(0-0,2)\end{array}$ & $\begin{array}{c}26.5 \\
(24-29,2)\end{array}$ & $\begin{array}{c}26.5 \\
(26-27,2)\end{array}$ & $\begin{array}{l}22 \\
(1)\end{array}$ & $\begin{array}{l}117 \\
(1)\end{array}$ \\
\hline \multicolumn{11}{|l|}{ Yonaguni-jima } \\
\hline Tabaru-gawa (28) & $12(113289)$ & $0.8-1.5$ & $10-23(7)$ & $55-64(4)$ & $\begin{array}{l}11.1 \pm 2.0 \\
(9-15,12)\end{array}$ & $\begin{array}{c}0 \pm 0 \\
(0-0,5)\end{array}$ & $\begin{array}{c}30.0 \pm 3.0 \\
(27-35,5)\end{array}$ & $\begin{array}{c}25.8 \pm 3.0 \\
(22-30,12)\end{array}$ & $\begin{array}{l}31.0 \pm 3.8 \\
(27-37,5)\end{array}$ & $\begin{array}{c}144.2 \pm 15.0 \\
(127-168,5)\end{array}$ \\
\hline \multicolumn{11}{|l|}{ Thailand } \\
\hline Songkhla Lagoon (29) & 1 (PMBC 29970) & 1.0 & 18 & 57 & 18 & 0 & 16 & 21 & 14 & 78 \\
\hline
\end{tabular}

${ }^{1}$ Corresponding to site numbers in Fig. 1 and Table $1 .{ }^{2}$ Catalogue numbers for the specimen depository in NSMT-Pol except for PMBC 29970 for a Thailand specimen. ${ }^{3}$ Data of only complete specimens. Range $(n) .{ }^{4}$ Chaetigrs with neuropodial postchaetal lobe. Mean \pm SD $(\operatorname{range}, n) .{ }^{5}$ Mean \pm SD (range, $n$ ). ${ }^{6}$ Larger value at a left or right side. ${ }^{7}$ Type locality. 


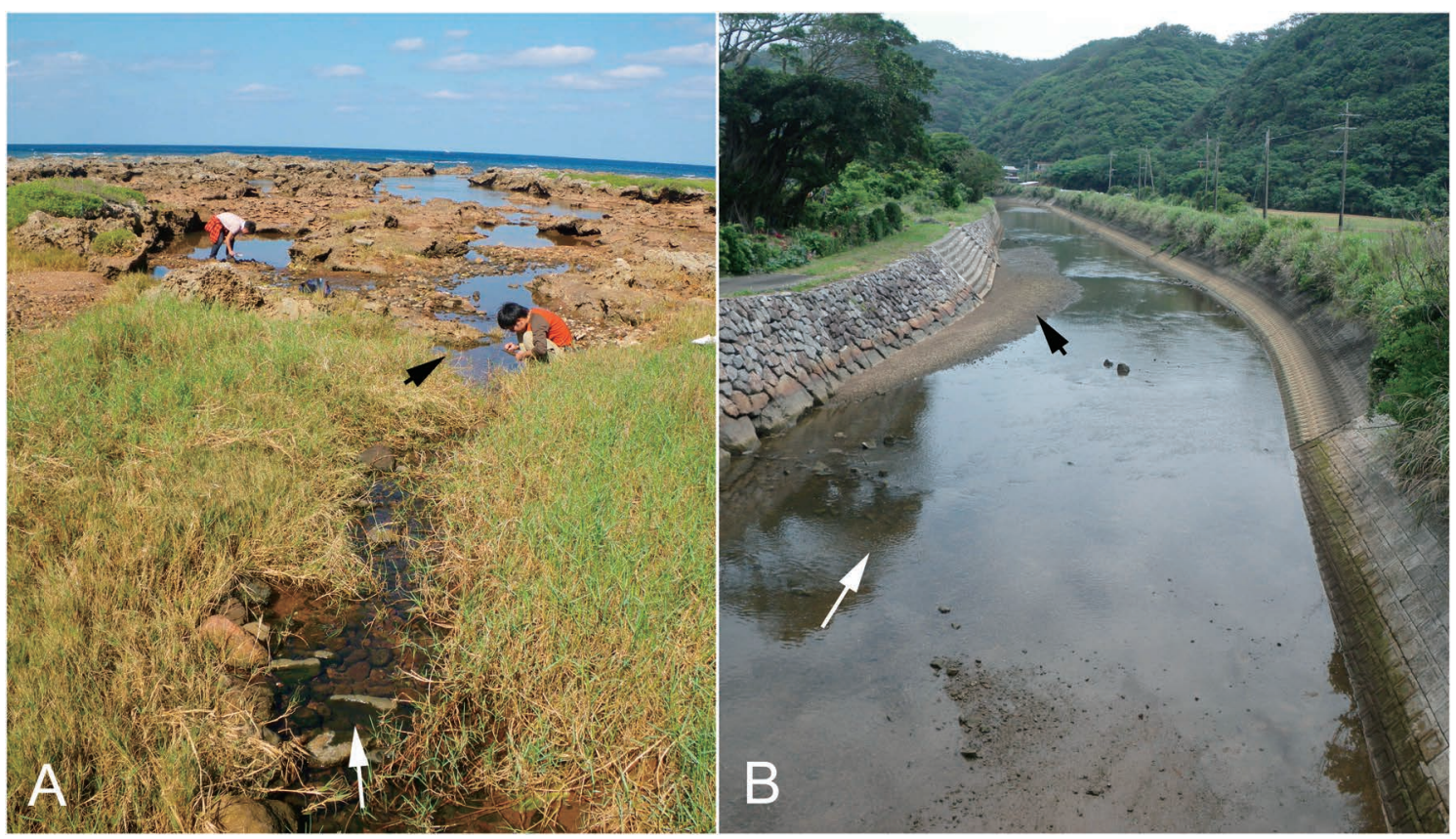

Fig. 2. Two types of habitats of Composetia in the Ryukyu Islands, southern Japan. A, habitat of the stream type on the uplifted coral reefs at East of Mifuga in Kume-jima island (No. 19 in Fig. 1 and Table 1), where C. kumensis was found; B, habitat of the usual river type in the uppermost estuary in Yani-gawa river in Amami-oshima island (No. 1 in Fig. 1 and Table 1), where C. tokashikiensis was found. Black arrows indicate the habitats of Composetia. White arrows indicate freshwater flow.
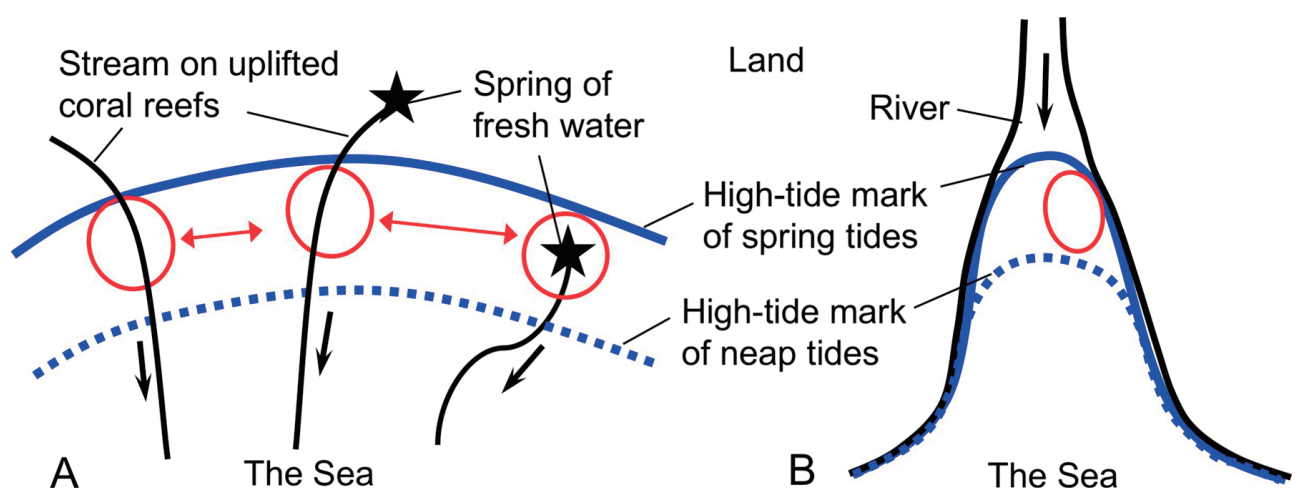

Fig. 3. Schematic diagram of two types of habitats of Composetia in the Ryukyu Islands, southern Japan. A, habitat of the stream type; B, habitat of the usual river type. In both types, the habitats are located in an uppermost narrow area between the high tide marks of spring tides and neap tides, in small estuaries of streams (A) or rivers (B). Red circles, habitats of Composetia; red arrows, probable dispersal of the young or adults; black arrows, freshwater flow.

island and Kikai-jima island; six individuals from Oura-gawa and four individuals from Teima-gawa belonged to four $(\mathrm{M}, \mathrm{N}, \mathrm{O}, \mathrm{P})$ and four $(\mathrm{M}, \mathrm{Q}, \mathrm{R}, \mathrm{S})$ haplotypes, respectively in the Oura Bay, while all of seven individuals from a single stream in Kikai-jima island belonged to a single haplotype (L); the genetic divergence was small among all of the eight haplotypes found in Okinawa-jima and Kikai-jima islands (Fig. 4; Table 4). While the Bayesian and ML analyses supported the genetic similarities of the haplotypes from the northern and southern areas of Kume-jima island with a relatively high posterior probability (0.98) and a moderate bootstrap (51\%) values, the MP and NJ analyses did not support such similarities (Fig. 4).

Allometry in Composetia kumensis. The body length
(BL $\mathrm{mm}$ ) was correlated with the body width (BW $\mathrm{mm}$ ) according to the following regression formula (Fig. 5A): $\mathrm{BL}=13.7 \quad \mathrm{BW}-0.16 \quad(r=0.70, \quad P<0.0001, \quad n=132)$. Total chaetiger number $(\mathrm{CN})$ was also correlated with $\mathrm{BW}$, according to the following regression formula (Fig. 5B): $\mathrm{CN}=22.2 \mathrm{BW}+28.4 \quad(r=0.61, P<0.0001, n=126)$. Maximum body sizes were $24 \mathrm{~mm}$ in $\mathrm{BL}, 1.5 \mathrm{~mm}$ in $\mathrm{BW}$, and 64 in $\mathrm{CN}$.

Total paragnath number (PN) was not significantly correlated with BW $(r=0.14, P=0.08, n=165)$ (Fig. $5 \mathrm{C})$.

Number of anterior chaetigers with a neuropodial postchaetal lobe (AC) was correlated with $\mathrm{BW}$, according to the following regression formula in smaller specimens $(1.0 \mathrm{~mm}$ or less in BW) (Fig. 5D): $\mathrm{AC}=6.2 \mathrm{BW}+3.1 \quad(r=0.37$, 
Table 4. Composition of 25 haplotypes obtained from 21 localities of two species of Composetia.

\begin{tabular}{|c|c|c|c|c|c|c|c|c|c|c|c|c|c|c|c|c|c|c|c|c|c|c|c|c|c|c|}
\hline \multirow{2}{*}{ Species } & \multirow{2}{*}{ Locality (site number) ${ }^{1}$} & \multicolumn{25}{|c|}{ No. of individuals with each haplotype } \\
\hline & & I & $\mathrm{J}$ & $\mathrm{K}$ & $\mathrm{L}$ & M & $\mathrm{N}$ & $\mathrm{O}$ & $\mathrm{P}$ & Q & $\mathrm{R}$ & $\mathrm{s}$ & A & B & $\mathrm{C}$ & $\mathrm{D}$ & $\mathrm{E}$ & $\mathrm{F}$ & G & $\mathrm{H}$ & $\mathrm{T}$ & $\mathrm{U}$ & $\mathrm{V}$ & $\mathrm{W}$ & $\mathrm{x}$ & $\mathrm{Y}$ \\
\hline \multirow[t]{8}{*}{ C. kumensis } & Urabaru-gawa, Kikai-jima $(8)^{2}$ & & & & 7 & & & & & & & & & & & & & & & & & & & & & \\
\hline & Oura-gawa, Okinawa-jima $(12)^{3}$ & & & & & 3 & 1 & 1 & 1 & & & & & & & & & & & & & & & & & \\
\hline & Teima-gawa, Okinawa-jima $(14)^{3}$ & & & & & 1 & & & & 1 & 1 & 1 & & & & & & & & & & & & & & \\
\hline & Hiyajou, Kume-jima $(17)^{2}$ & 3 & & & & & & & & & & & & & & & & & & & & & & & & \\
\hline & Tachijami, Kume-jima $(18)^{2}$ & 3 & & & & & & & & & & & & & & & & & & & & & & & & \\
\hline & East of Mifuga, Kume-jima $(19)^{2}$ & 3 & & & & & & & & & & & & & & & & & & & & & & & & \\
\hline & Gushicha Gusuku, Kume-jima $(20)^{2}$ & 3 & & & & & & & & & & & & & & & & & & & & & & & & \\
\hline & Shirase-gawa, Kume-jima (21) & & 1 & 1 & & & & & & & & & & & & & & & & & & & & & & \\
\hline \multirow[t]{15}{*}{ C. tokashikiensis } & Yani-gawa, Amami-oshima (1) & & & & & & & & & & & & 1 & & & & & & & & & & & & & \\
\hline & Mae-kawa, Amami-oshima (2) & & & & & & & & & & & & & & 1 & & & & & & & & & & & \\
\hline & Ura, Amami-oshima (4) & & & & & & & & & & & & & & & & & 1 & & & & & & & & \\
\hline & Nakata-gawa, Kakeroma-jima (6) & & & & & & & & & & & & & & & 1 & & & & & & & & & & \\
\hline & Yamada-gawa, Kakeroma-jima (7) & & & & & & & & & & & & & 2 & & & & & & & & & & & & \\
\hline & Gabui, Okinawa-jima (10) & & & & & & & & & & & & & & & & 1 & & & & & & & & & \\
\hline & Oura-gawa, Okinawa-jima $(12)^{3}$ & & & & & & & & & & & & & & 1 & & & & & & & & & & & \\
\hline & Teima-gawa, Okinawa-jima $(14)^{3}$ & & & & & & & & & & & & & & 7 & & & & & & & & & & & \\
\hline & Tokashiki-gawa, Tokashiki-jima (16) & & & & & & & & & & & & & & & & 1 & & 1 & 7 & & & & & & \\
\hline & Hirakubo, Ishigaki-jima (22) & & & & & & & & & & & & & & 1 & & & & & & & 1 & 1 & & & \\
\hline & Ohura-gawa, Ishigaki-jima (24) & & & & & & & & & & & & & & 3 & & & & & & & & & & & \\
\hline & Inoda-gawa, Ishigaki-jima (25) & & & & & & & & & & & & & & & & & & & & & & & & & 3 \\
\hline & Touro-gawa, Ishigaki-jima (26) & & & & & & & & & & & & & & & & & & & & & & & 1 & 3 & \\
\hline & Anparu, Ishigaki-jima (27) & & & & & & & & & & & & & & 1 & & & & & & 1 & & & & & \\
\hline & Tabaru-gawa, Yonaguni-jima(28) & & & & & & & & & & & & & & & & & & & & & & & 3 & & \\
\hline
\end{tabular}

${ }^{1}$ Corresponding to site numbers in Fig. 1 and Table $1 .{ }^{2}$ Habitat type A. ${ }^{3}$ In the two rivers, Oura-gawa and Teima-gawa, both of the two species coexisted.

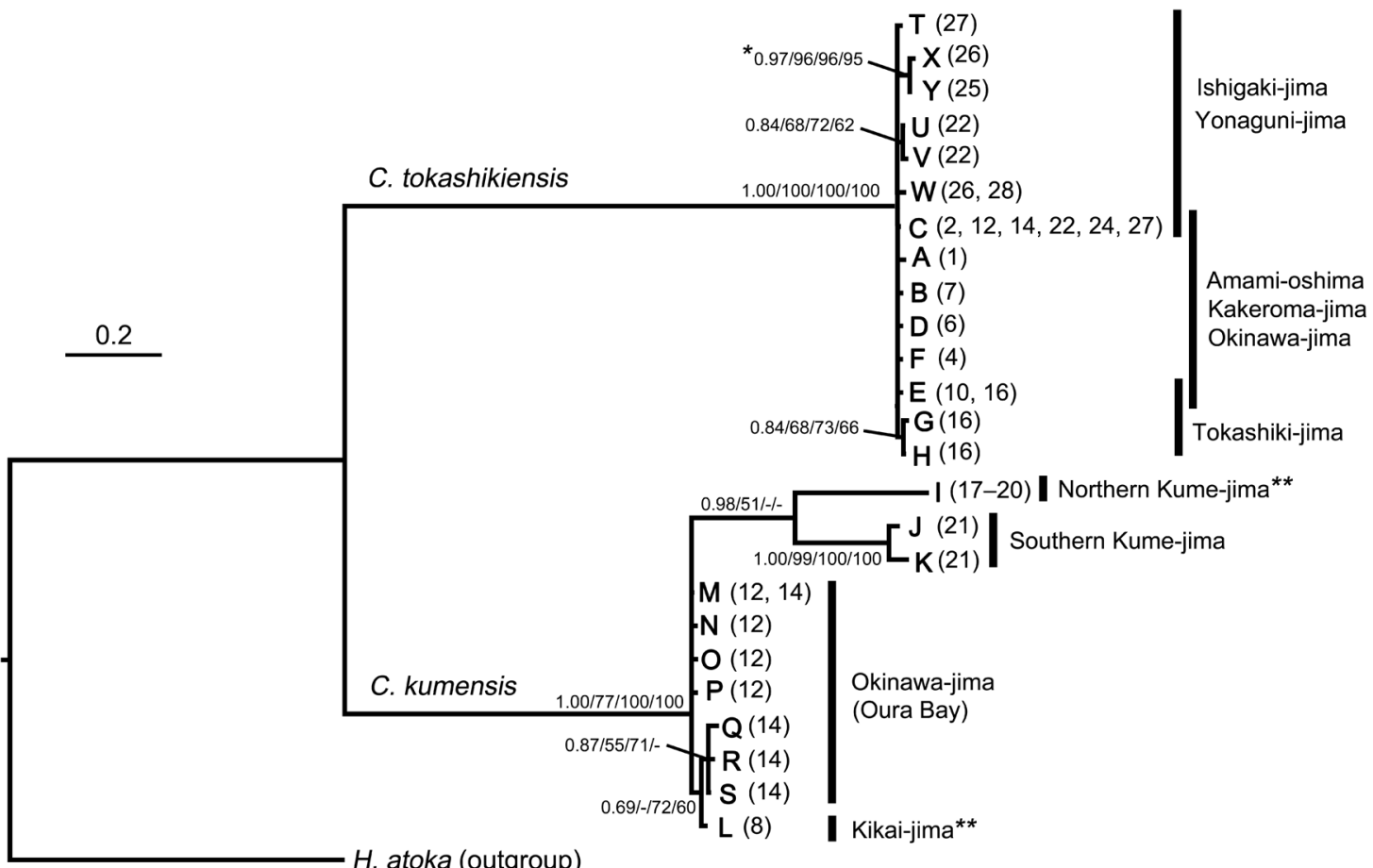

Fig. 4. Bayesian tree constructed from COI haplotypes (694 bp) of Composetia kumensis ( $n=11)$ and C. tokashikiensis (14), obtained from 8 and 15 localities, respectively. A-Y, haplotypes of mitochondrial COI gene; (1-28), site numbers corresponding to those in Fig. 1 and Tables 1-4. *Bayesian posterior probability and bootstrap values of ML, MP, and NJ, represented as Bayesian/ML/MP/NJ. **Habitats of the stream type. 

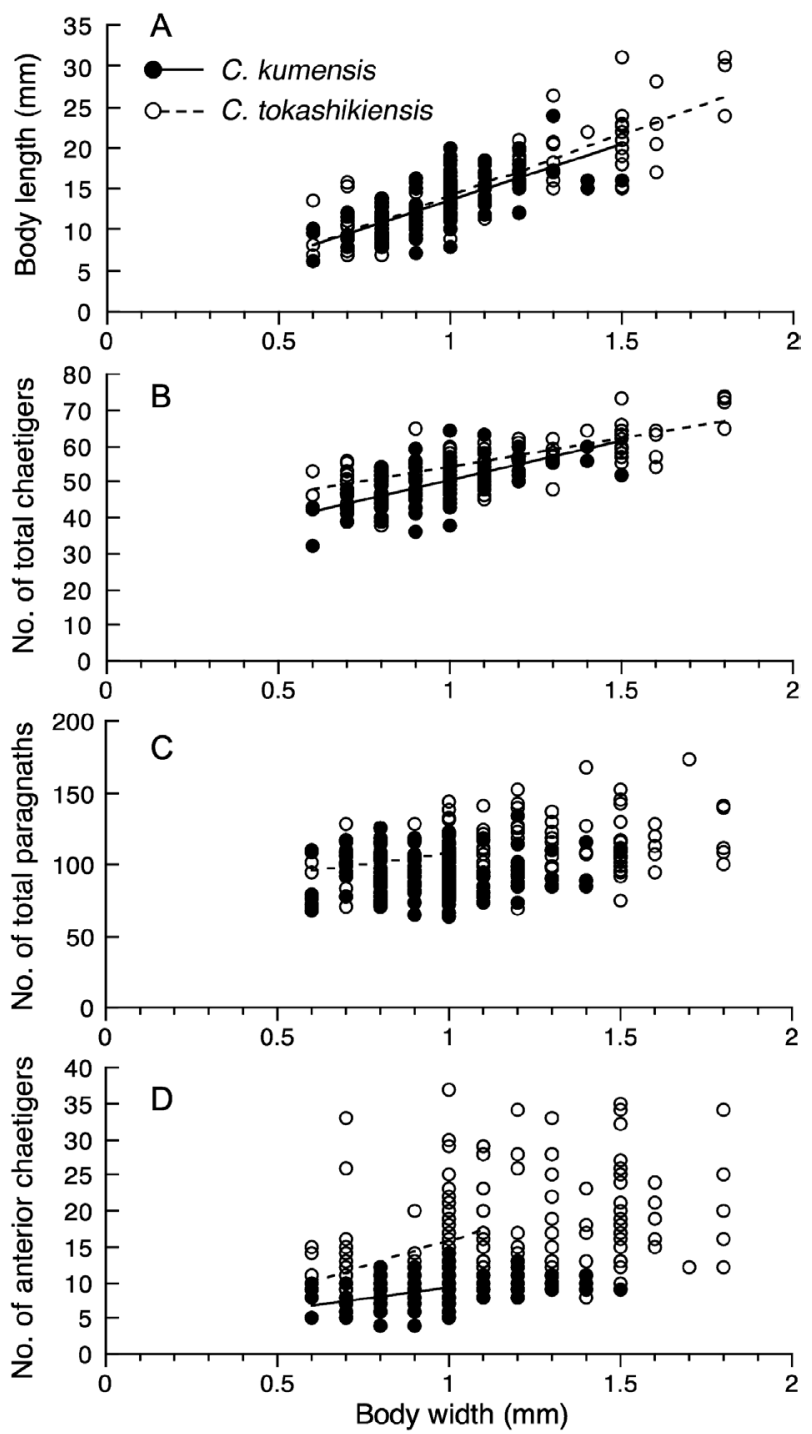

Fig. 5. Relationships between body width and body length (A), number of total chaetigers (B), number of total paragnaths (C) and number of anterior chaetigers (D) in two species of Composetia. Regression lines with a statistically significant correlation are also shown.

$P<0.0001, n=128)$, whereas it was not significantly correlated with $\mathrm{BW}$ in larger specimens $(1.0 \mathrm{~mm}$ or more in $\mathrm{BW})$ $(r=0.06, P=0.57, n=102)$.

Allometry in Composetia tokashikiensis. The body length (BL mm) was correlated with the body width (BW $\mathrm{mm}$ ) according to the following regression formula (Fig. 5A): $\mathrm{BL}=15.0 \mathrm{BW}-0.9(r=0.85, P<0.0001, n=121)$. Total chaetiger number $(\mathrm{CN})$ was also correlated with $\mathrm{BW}$, according to the following regression formula (Fig. 5B): $\mathrm{CN}=15.5 \mathrm{BW}+38.5(r=0.71, P<0.0001, n=111)$. Maximum body sizes were $31 \mathrm{~mm}$ in $\mathrm{BL}, 1.8 \mathrm{~mm}$ in $\mathrm{BW}$, and 74 in $\mathrm{CN}$.

Total paragnath number (PN) was correlated with BW, according to the following regression formula in smaller specimens ( $1.0 \mathrm{~mm}$ or less in BW) (Fig. $5 \mathrm{C}$ ): $\mathrm{PN}=32.1$ $\mathrm{BW}+76.3(r=0.29, P=0.01, n=78)$, whereas it was not significantly correlated with BW in larger specimens $(1.0 \mathrm{~mm}$
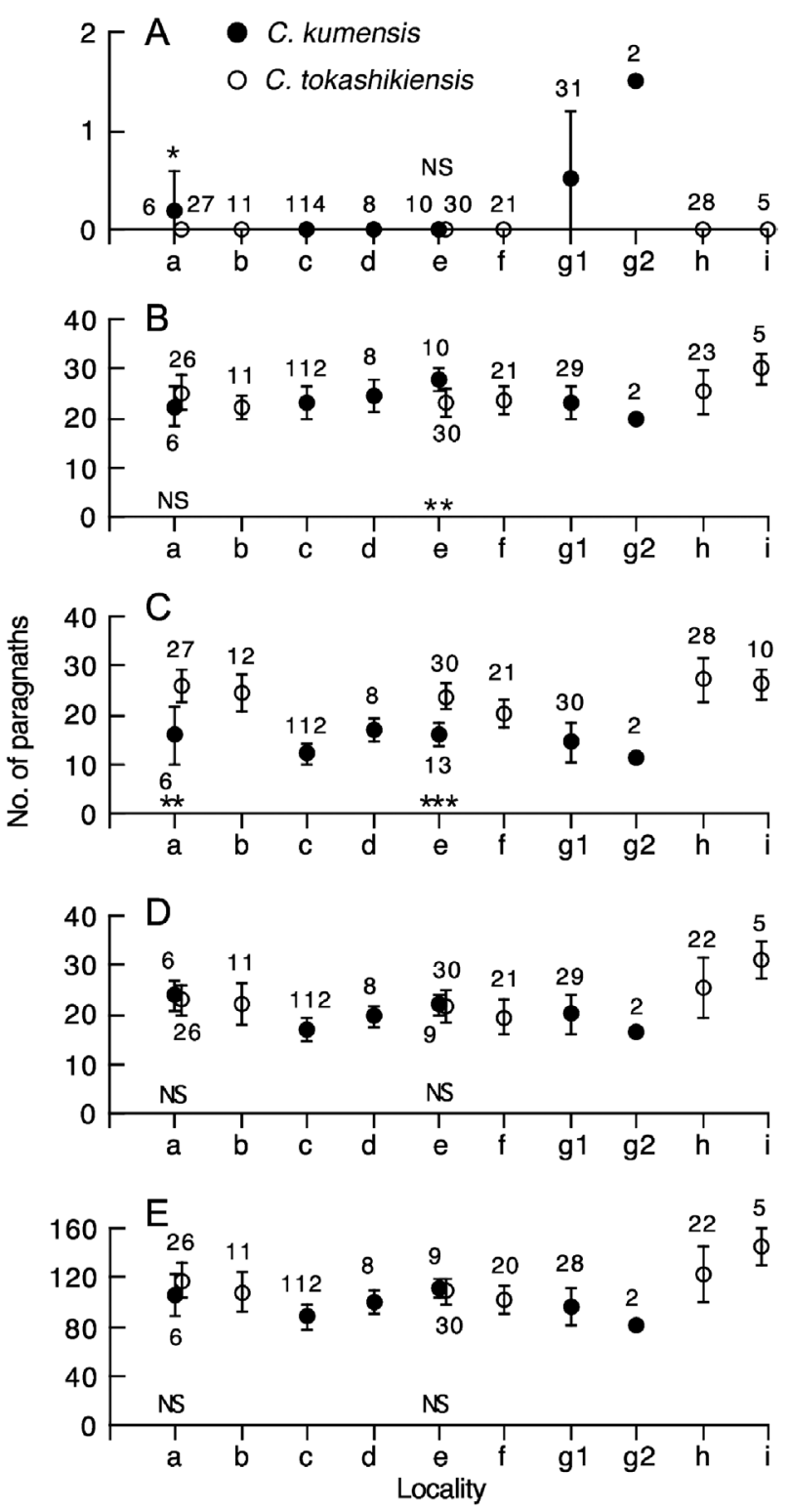

Fig. 6. Geographical variation in the number of paragnaths on areas I (A), II (B), III (C), IV (D) and all areas (E) in proboscis of two species of Composetia collected from 10 localities in the middle and southern Ryukyu Islands, southern Japan. Numbers of paragnaths on the areas II and IV were represented by the larger number in either right or left sides. Average and SD bar are shown in each locality. The number on each datum indicates sample size. The localities are arranged from north to south. Difference of mean numbers between the sympatric populations of C. kumensis and C. tokashikiensis was not significant (NS, $P>0.05$ ) or significant $\left({ }^{*} P<0.05\right.$; ** $P<0.001$; *** $P<0.0001)$ by the Mann-Whitney U-test. a, Amami-oshima (site numbers in Fig. 1 and Table 1: 1-5); b, Kakeromajima (6, 7); c, Kikai-jima (8); d, Tokuno-shima (9); e, Okinawa-jima (10-15); f, Tokashiki-jima (16), g1, northern Kume-jima (17-20); g2, southern Kume-jima (21); h, Ishigaki-jima (22-27); i, Yonaguni-jima (28).

or more in BW) $(r=0.16, P=0.08, n=115)$.

Number of anterior chaetigers with a neuropodial postchaetal lobe (AC) was correlated with BW, according to the following regression formula in smaller specimens $(1.1 \mathrm{~mm}$ or less in BW) (Fig. 5D): $\mathrm{AC}=14.0 \mathrm{BW}+1.8 \quad(r=0.33$, 
$P=0.0005, n=105)$, whereas it was not significantly correlated with $\mathrm{BW}$ in larger specimens $(1.1 \mathrm{~mm}$ or more in $\mathrm{BW})$ $(r=0.11, P=0.3, n=86)$.

Variation of paragnath numbers in the two species of Composetia. Paragnath numbers on the areas I-IV in all specimens of C. kumensis in 10 sites on five islands, and those of C. tokashikiensis in 20 sites on six islands are summarized in Tables 2 and 3, respectively. For the present analysis on the interspecific and interpopulation variations, data from all sites on each island were pooled as a single population, except for those in Kume-jima island, where data from a site (Shirase-gawa river) in the southern area were separated from those from the other four sites in the northern area, because a marked genetic differentiation was detected between the two areas, as mentioned above. Data of small individuals of $C$. tokashikiensis less than $1 \mathrm{~mm}$ in BW were not used, because their paragnath numbers increased depending on the growth of their body size, as mentioned above (Fig. 5C).

The paragnath numbers are compared between populations on nine islands in the Ryukyu Islands (Fig. 6). The area I lacked paragnath completely in all populations of C. tokashikiensis, whereas one or two paragnaths present in some individuals of C. kumensis in two islands: rarely present in Amami-oshima island, and often present in Kume-jima island, though completely absent in Kikai-jima, Tokuno-shima and Okinawa-jima islands (Fig. 6A). On the area II, the mean paragnath numbers varied slightly between populations within 20-30 in both species (Fig. 6B); the ranges in C. kumensis (16-36) and C. tokashikiensis (16-35) were almost the same. On the area III, the mean paragnath numbers in all seven populations of C. tokashikiensis (20-27) were larger than those in all six populations of C. kumensis (11-17), though the ranges in C. tokashikiensis (14-37) and C. kumensis (7-25) overlapped; the interspecific differences were significant in two combinations, in which both of the two species occurred in the same islands (Amami-oshima and Okinawa-jima) (Mann-Whitney U-test, $P<0.001$ ) (Fig. $6 \mathrm{C})$. On the area IV, the mean paragnath numbers varied between populations within 19-31 in C. tokashikiensis, and within 17-24 in C. kumensis (Fig. 6D), with the ranges in C. tokashikiensis (10-38) and C. kumensis (11-28) overlapping; the interspecific differences were not significant in the two combinations. As for the total paragnath numbers, the mean values varied between populations within 101-144 in C. tokashikiensis, and within 81-111 in C. kumensis (Fig. 6E), with the ranges in C. tokashikiensis (69-173) and C. kumensis (64-134) overlapping; the interspecific differences were not significant in the two combinations.

Variation of numbers of anterior chaetigers in the two species of Composetia. Numbers of anterior chaetigers having neuropodial postchaetal lobes in all specimens of $C$. kumensis in 10 sites on five islands, and those of C. tokashikiensis in 20 sites on six islands are summarized in Tables 2 and 3, respectively.

For the present analysis, data from all sites on each island were pooled as a single population, as mentioned above for the analysis of paragnath numbers, with the exception of

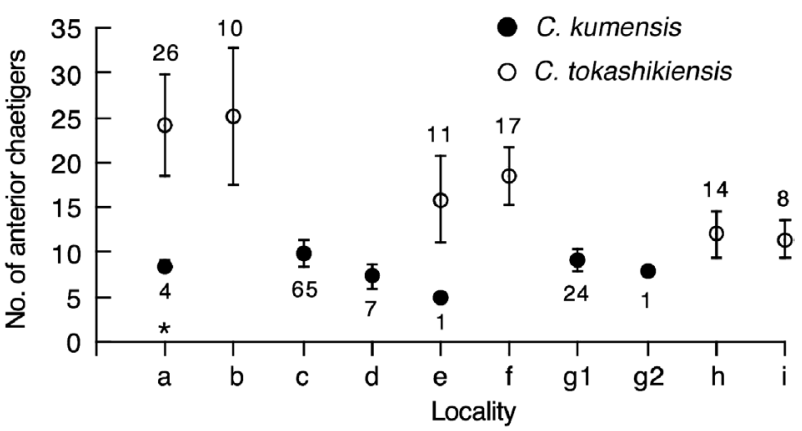

Fig. 7. Geographical variation in the number of anterior chaetigers of two species of Composetia collected from 10 localities in the middle and southern Ryukyu Islands, southern Japan. Average and SD bar are shown in each locality. The number on each datum indicates sample size. The localities are arranged from north to south. Abbreviations of localities are the same as in Fig. 6. *Difference of mean numbers between the sympatric populations of $C$. kumensis and C. tokashikiensis was significant (Mann-Whitney Utest, $P=0.0016$ )

those in Kume-jima island. Data for small individuals of C. kumensis less than $1 \mathrm{~mm}$ in $\mathrm{BW}$, and for C. tokashikiensis less than $1.1 \mathrm{~mm}$ in BW were not used, because their numbers of anterior chaetigers increased depending on the growth of their body size, as mentioned above (Fig. 5D).

The numbers of anterior chaetigers are compared between populations on nine islands in the Ryukyu Islands (Fig. 7). The mean values in all of seven populations of $C$. tokashikiensis (11-25) were larger than those in all of six populations of $C$. kumensis (5-10), though the ranges in $C$. tokashikiensis (8-35) and C. kumensis (5-14) overlapped; the interspecific difference was significant in a combination, in which both species occurred in the same island (Amamioshima) (Mann-Whitney U-test, $P=0.0016$ ). In C. tokashikiensis, a latitudinal cline that the number of anterior chaetigers decreases in southern populations was recognized.

Reproductive characteristics of the two species of Composetia. In C. kumensis, the largest coelomic oocyte of $330 \mu \mathrm{m}$ in diameter was found in a female collected from Urabaru-gawa river in Kikai-jima island on 28 November 2009. In the type locality of this species (Gushicha Gusku in Kume-jima island), the largest oocyte of $250 \mu \mathrm{m}$ in diameter was found in a female collected on 23 March 2007 (Sato 2020).

In C. tokashikiensis, the largest coelomic oocyte of $250 \mu \mathrm{m}$ in diameter was found in a female collected from Okukubigawa river in Okinawa-jima island on 10 September 2004. The same maximum diameter of oocytes was also recorded in a paratype female collected from Tokashiki-gawa in Tokashiki-jima island on 20 November 1991 (Sato 2020).

In both species, none of specimens show any epitokous metamorphosis.

New record of Composetia tokashikiensis from Thailand. A single specimen collected from an estuary in Thailand was identified as C. tokashikiensis. A morphological description of this specimen is provided here as a new record of this species from Thailand: 

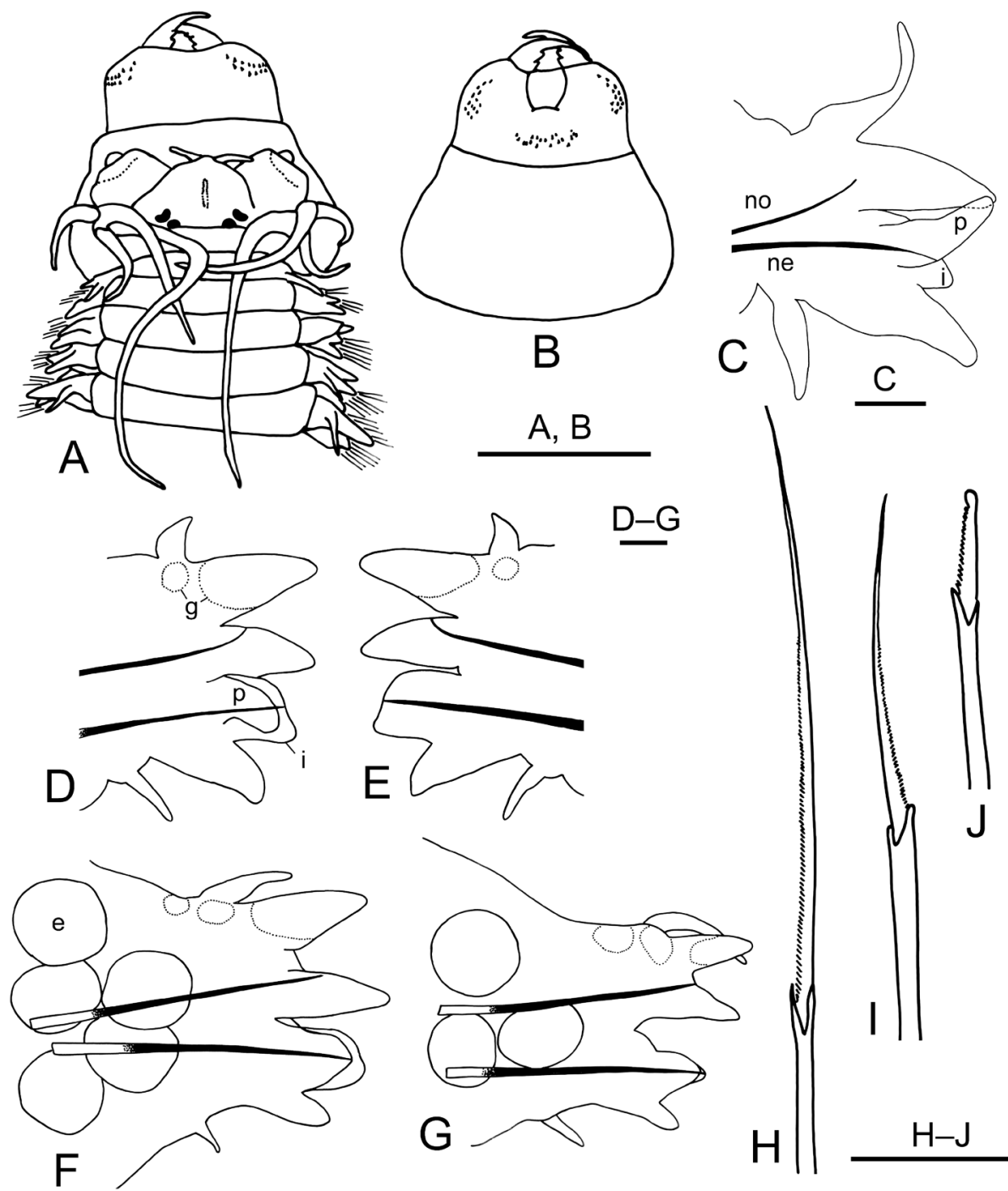

$\underline{D-G}$
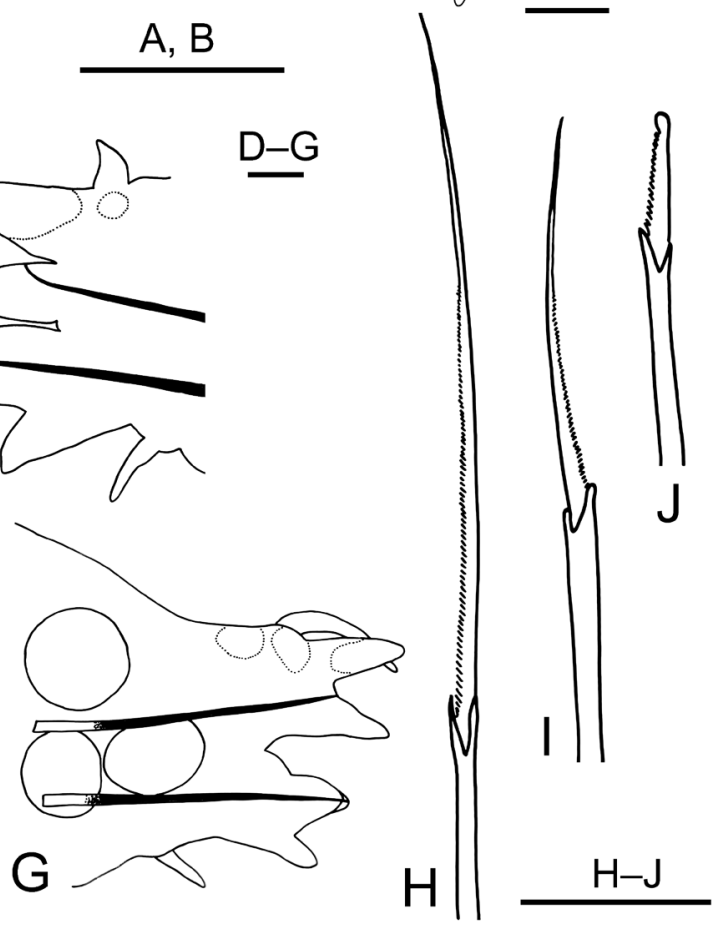

Fig. 8. Composetia tokashikiensis Sato, 2020, from Songkhla Lagoon, Thailand (PMBC 29970). A, dorsal views of the anterior body with the everted proboscis; B, ventral view of the everted proboscis; C, posterior view of right parapodium 1; D, posterior view of right parapodium 5; E, anterior view of right parapodium 5; F, posterior view of right parapodium 20. G, posterior view of right parapodium 46 . $\mathrm{H}$, homogomph spiniger from upper neurochaetae in parapodium $1 ; \mathrm{I}$, heterogomph spiniger with short blade from lower neurochaetae (lower position) in parapodium 5; J, heterogomph falciger from upper neurochaetae in parapodium 1. Abbreviations: g, glandular patch; i, neuropodial inferior lobe; ne, neuroacicula; no, notoacicula; p, neuropodial postchaetal lobe. Scale bars: $1 \mathrm{~mm}(\mathrm{~A}, \mathrm{~B}) ; 0.1 \mathrm{~mm}(\mathrm{C}-\mathrm{G}) ; 0.05 \mathrm{~mm}(\mathrm{H}-\mathrm{J})$.

\section{Composetia tokashikiensis Sato, 2020}

(Fig. 8)

Composetia tokashikiensis Sato, 2020: 17-20, figs 4B, 6-8. Composetia sp. A: Sato and Sakaguchi 2016: 85.

Composetia sp. 1: Sato 2017: 483.

Materials examined. One single female (PMBC29970), the lower reaches of Songkhla Lagoon $\left(7^{\circ} 10^{\prime} 37.4^{\prime \prime} \mathrm{N}\right.$, $100^{\circ} 32^{\prime} 26.2^{\prime \prime} \mathrm{E}$ ), southern Thailand, 19 November 2008 , coll. S. Angsupanich (Fig. 1; Table 1).

Description. Complete individual, $18 \mathrm{~mm} \mathrm{BL}, 1.0 \mathrm{~mm}$ BW, with 57 chaetigers. Colour in preserved specimen whitish cream with brownish pigmentation in anterior dorsum.

Prostomium pear-shaped (Fig. 8A). Antennae short, tapered. Palps with massive palpophores and short subconical palpostyles. Two pairs of eyes arranged trapezoidally, ante- rior pair reniform, more separated and larger than posterior pair; posterior pair round. Mid-longitudinal white slit present on dorsal anterior surface of prostomium.

Apodous segment (peristomium) with four pairs of tentacular cirri of unequal length; posterior dorsal tentacular cirri longest, reaching back to chaetigers 11 .

Proboscis with pair of amber jaws, each with eight marked teeth. Brown paragnaths present only on maxillary ring (Fig. 8A, B). Paragnath numbers on each area as follows: area I: 0; area II: 13 on left and 16 on right in two arched rows, total 29; area III: 21 in wide patch; area IV: 14 on each side in triangular patch, total 28 . Oral ring greatly enlarged into trapezoidal shape in full-everted proboscis, without any paragnaths or papillae.

Sub-biramous parapodia of first 2 chaetigers with thin notoacicula (Fig. 8C). Notopodial dorsal ligule conical with tapering tip throughout. Both notopodial prechaetal lobe 
and notoacicular process absent throughout (Fig. 8D-G). Notopodial ventral ligule conical with tapering tip throughout, almost subequal to notopodial dorsal ligule. Dorsal cirri slender in most chaetigers (relatively thick in chaetiger 5), tapering, as long as or shorter than notopodial dorsal ligule throughout (Fig. 8C-G). Three (rarely two) whitish glandular patches present on dorsal edge of notopodia; distalmost glandular patch larger than others, covering whole conical projection of notopodial dorsal ligule throughout (Fig. 8D-G).

Neuropodial postchaetal lobe with tapering tip present in first 18 chaetigers (Fig. 8C, D), absent in following chaetigers (Fig. 8F, G). Superior lobe in acicular ligule absent throughout. Inferior lobe conical in anterior parapodia, diminishing in middle parapodia, and absent in posterior parapodia. Ventral ligule conical with tapering tip throughout. Ventral cirrus slender with tapering tip.

Notochaetae all homogomph spinigers, having long blades with finely serrated edge; up to 8 spinigers present.

Upper neurochaetae at superior/anterior position in anterior chaetigers (around first 20 chaetigers) consisting of heterogomph spinigers with short finely-serrated blades $(3,7$, and 0 spinigers present in chaetigers 1,5 , and 20 , respectively), and few heterogomph falcigers with short finely-serrated blades (Fig. 8J) (1, 0, and 2 falcigers present in chaetigers 1 , 5 , and 20, respectively). Upper neurochaetae at superior/anterior position in posterior chaetigers consisting of only few heterogomph falcigers $(0,2$, and 2 falcigers present in chaetigers 44,46 , and 48 , respectively), lacking heterogomph spinigers.

Upper neurochaetae at posterior position consisting of homogomph spinigers with long finely-serrated blades (Fig. $8 \mathrm{H}$ ) throughout (up to 6 spinigers present).

Lower neurochaetae in anterior chaetigers consisting of many heterogomph spinigers with finely-serrated blades $(12,13$ and 4 spinigers present in chaetigers 1,5 and 20, respectively), and few heterogomph falcigers with short serrated blades $(0,1$ and 3 falcigers present in chaetigers 1, 5 and 20, respectively); spinigers with long blades located at posterior position; spinigers with short blades (Fig. 8I) and falcigers located at inferior/anterior position. Lower neurochaetae in posterior chaetigers consisting of several heterogomph spinigers at posterior position ( 5,5 and 4 spinigers present in chaetigers 44,46 and 48 , respectively), and few heterogomph falcigers at inferior/anterior position $(0,1$ and 1 falcigers present in chaetigers 44,46 and 48 , respectively).

The coelom filled with oocytes of $180-200 \mu \mathrm{m}$ in diameter.

Habitat. Shallow subtidal bottom in the coast of the lower reaches of Songkhla Lagoon, where salinity widely ranges from 1 to 33 psu depending on variable seasonal rainfall; in general, about 1-10 psu in November to March, about $10-20$ psu in April to July, about 20-33 in August to October (Angsupanich and Rakkheaw 1997; S. Angsupanich, unpublished data).

Geographical distribution. The middle and southern Ryukyu Islands in southern Japan (north to Amami-oshima, south to Yonagni-jima), the coast of South China Sea in southern Thailand (Fig. 1).

Remarks. The morphological characteristics of the present Thailand specimen agreed well with those of the original description of C. tokashikiensis by Sato (2020) and also of many additional non-type specimens of this species collected from the Ryukyu Islands, southern Japan in the present study. All of the numerical characteristics (BW, BL, numbers of total chaetigers, anterior chaetigers, and paragnaths) of the Thailand specimen were within the ranges of their variations, which were mentioned above for the Japanese populations (Figs 6, 7; Table 3).

However, the notoacicular process was absent in the Thailand specimen that was an ovigerous female with BW of $1.0 \mathrm{~mm}$, whereas it was present in a few parapodia in chaetigers 5-10 in holotype and some large paratypes of C. tokashikiensis with a BW of $1.5 \mathrm{~mm}$ or more (Sato 2020). In the present study, the presence and absence of the notoacicular process was regarded as an intraspecific variation depending on the body size.

\section{Discussion}

Geographical distribution and habitat preference. The present study shows that Composetia tokashikiensis is widely distributed in the middle and southern Ryukyu Islands, from north to Amami-oshima island to south to Yonaguni-jima island, whereas C. kumensis is distributed in a relatively narrow area in the middle Ryukyu Islands, from north to Kikai-jima island to south to Kume-jima island. The finding of C. tokashikiensis in Thailand suggests that this species is widely distributed in tropical and subtropical estuaries in western Pacific Ocean.

Habitats of both species were located in a narrow area in the uppermost reaches of small-scale estuaries in islands except for in the lower reaches of Songkhla Lagoon in Thailand. The uppermost reaches of small-scale estuaries are characterized by the extreme fluctuation of salinity from usual fresh water to sometimes full-strength seawater, because the freshwater inflow is limited there. The extreme fluctuation of salinity from 1 to 33 psu is also known in the habitat of C. tokashikiensis in Songkhla Lagoon in Thailand, although it is a large-scale lagoon (Angsupanich and Rakkheaw 1997; S. Angsupanich, unpublished data).

Such an extreme fluctuation of salinity seems to be physiologically severe for many benthic invertebrates to survive. Therefore, the uppermost reaches of small-scale estuaries in islands are inhabited by only a limited number of macrobenthic invertebrates, which have gained a tolerance to the wide range of salinity, with a benefit of low interspecific competition. The two species of Composetia (C. kumensis in particular), which dominate in these habitats with no or few associated macrobenthic fauna, seem to be representative of rare successful benthos well adapted to the extreme fluctuation of salinity. Adult worms of C. kumensis collected there can survive under either conditions of the constant fresh water ( 0.4 psu in salinity) or seawater ( $25 \mathrm{psu}$ ) in the laboratory for more than two months (our unpublished data). 
In the most habitats, C. kumensis or C. tokashikiensis occurred exclusively. Whereas the former was found from habitats of both the stream and usual river types, the latter occurred only in habitats of the usual river type. These facts suggest that there may be some difference in habitat preference between the two species.

It is unknown why both species can coexist sympatrically in the two neighboring rivers (Oura-gawa and Teima-gawa) in Oura Bay in the east coast of Okinawa-jima island. The Oura Bay is well known as a rare place where natural environments including the coral-reef, mangrove and surrounding terrestrial forest ecosystems have been exceptionally well preserved, keeping extremely high marine species diversity, with 5334 species recorded in the coastal macro fauna and flora (Diving Team Snack Snufkin 2015; Obuchi 2015); the rich fauna of riverine fish are also known in Teima-gawa river (191 species from estuarine and fresh waters; Maeda and Tachihara 2006) and Oura-gawa river (110 species from the estuary; Uehara and Nishijima 2003).

Life history and dispersal ability. In general, the size of fully-mature eggs is related to the life-history pattern in macrobenthic invertebrates. Species which produce large numbers of smaller eggs have a longer pelagic larval phase in their life history and can disperse extensively by means of planktic larvae, whereas species which produce small numbers of larger eggs have a shorter or no pelagic larval phase with their larval dispersal limited (Thorson 1950; Schroeder and Hermans 1975; Sato 1999). Though the exact size of fully-mature eggs of the two species of Composetia have not yet been determined, our data on the maximum diameter of coelomic oocytes ( $330 \mu \mathrm{m}$ for C. kumensis and $250 \mu \mathrm{m}$ for $C$. tokashikiensis) indicate that both of them produce relatively large mature eggs, which are comparative to those of $\mathrm{He}$ diste atoka (200-250 $\mu \mathrm{m}$ in diameter, developing into benthic juveniles without pelagic larval life) (Sato and Tsuchiya 1991; Sato 1999) and Perinereis spp. [as P. brevicirris (Grube, 1869), $P$. nuntia var. brevicirris or $P$. nuntia var. vallata (Grube, 1857), about $300 \mu \mathrm{m}$ in diameter, developing into planktic larvae with 1-week pelagic life] (Fukushima and Ishida 1966; Yoshida 1984) in a comparison within Nereididae. Therefore, it is probable that both of the two species of Composetia have also a life history with no or short pelagic larval phase, suggesting that their ability of larval dispersal beyond the sea is absent or very limited.

However, the two species of Composetia may be able to disperse long way from an estuary to another one beyond the sea at their juvenile or adult phases by means of floating and swimming behavior of juveniles, as reported in $\mathrm{H}$. atoka (Sato 1999; Kikuchi and Yasuda 2006) or riding of juveniles and adults on driftwoods, because their juveniles and adults seem to survive the travel through full-strength seawater as mentioned above.

Inter- and intra-specific differentiations in the Ryukyu Islands. Our result of molecular analyses shows the marked genetic differentiation between the two species of Composetia in the Ryukyu Islands, strongly supporting that they are clearly distinct species in spite of their morphological similarity (see below).
Marked intra-specific genetic differentiations were detected among populations of C. kumensis; three haplotypes (I, J, $\mathrm{K}$ ) in Kume-jima island were markedly differentiated from all of the other eight haplotypes in Okinawa-jima (Oura Bay) and Kikai-jima islands; furthermore, a single haplotype (I) in four neighboring populations in northern Kume-jima island was also largely differentiated from two haplotypes (J, $\mathrm{K})$ in the Shirase-gawa population in southern Kume-jima island. This result indicates that the northern Kume-jima and southern Kume-jima populations have been isolated without any gene flows for long time from each other and from the other populations in Okinawa-jima and Kikai-jima islands, depending on the probable poor ability of the larval dispersal in this species as mentioned above. The fact that only a single haplotype (I) was shared in four neighboring populations in habitats of the stream type in northern Kume-jima island (Fig. 1D) indicates the absence of isolation among these neighboring populations, which can be regarded as a single metapopulation; frequent gene flows seem to be maintained among them by migrating juveniles or adults during high tides at the extreme spring tides, when the neighboring habitats of the stream type are connected by flooding seawater (Fig. 3A).

The diversity of haplotypes in C. kumensis was the highest in two neighboring populations in habitats of the usual river type in Oura Bay in Okinawa-jima island; a total of seven haplotypes were found in a total of 10 individuals examined there. This fact suggests that a relatively large population may have been maintained for a long time there. Three haplotypes $(\mathrm{N}, \mathrm{O}, \mathrm{P})$ were endemic to Oura-gawa river, and other three haplotypes $(\mathrm{Q}, \mathrm{R}, \mathrm{S})$ were endemic to Teima-gawa river (Table 4, Fig. 4), with a single haplotype (M) common to both rivers. A slight genetic differentiation was detected between the Teima-gawa endemic haplotypes and the other ones. This fact suggests that gene flows have not been frequent even between the two neighboring populations in habitats of the usual river type within Oura Bay.

Only a single haplotype (L) was shared by all of seven individuals examined in a population in a habitat of the stream type in Urabaru-gawa river in Kikai-jima island, closely related to the Teima-gawa endemic haplotypes ( $Q$, R, S) (Fig. 4; Table 4). This fact suggests that the Urabarugawa population was originated from few successful founders that migrated beyond the sea (e.g., by means of juveniles or adults on driftwoods) from the Teima-gawa population or its unknown relatives somewhere in the eastern coast of Okinawa-jima island in a relatively recent age.

In the present study, habitats of the stream type were found from only two places for C. kumensis: a metapopulation in four neighboring streams in northern Kume-jima island, and the Urabaru-gawa population in Kikai-jima island. In both habitats, the genetic diversity was so low that only single haplotype was found in each habitat. This may be caused by a genetic drift associated with a bottleneck effect. The habitats of the stream type are characterized by a small population size within a small stream, and a location on the uplifted coral reefs, which faces the open sea. If the dispersal beyond the sea by means of juveniles or adults 
on driftwoods is effective, the migrating juveniles or adults would most easily reach the habitats of the stream type, where a single haplotype may be fixed easily by a genetic drift.

On the other hand, no marked genetic differentiation of haplotypes was detected among any local populations of $C$. tokashikiensis, though six haplotypes (T, U, V, W, X, Y) were endemic to the southern Ryukyu Islands (Ishigaki-jima and Yonaguni-jima islands), and other two haplotypes $(\mathrm{G}, \mathrm{H})$ were endemic to Tokashiki-gawa river in Tokashiki-jima island (Fig. 4; Table 4). This fact suggests that the dispersal ability of larvae, juveniles or adults is relatively high in comparison to that of C. kumensis. This idea is supported by the presence of the widespread haplotype $C$ that occurred throughout the range of this species in the Ryukyu Islands (from Amami-oshima to Ishigaki-jima islands).

In contrast to the high diversity of haplotypes of $\mathrm{C} . \mathrm{ku}$ mensis in two neighboring populations of Oura-gawa and Teima-gawa rivers in Oura Bay in Okinawa-jima island, only the single widespread haplotype $\mathrm{C}$ was found in a total of eight individuals of $C$. tokashikiensis there (Table 4). This fact suggests that C. tokashikiensis may have colonize there relatively recently.

As for morphological characteristics, we could detect somewhat differentiations between the two species in the following two characteristics: (1) the mean paragnath numbers on the area III (Fig. 6C), and (2) the mean numbers of anterior chaetigers with a neuropodial postchaetal lobe (Fig. 7). In both characteristics, the values of all populations of $C$. tokashikiensis were larger than those of $C$. kumensis, though their ranges of the two species overlapped. Therefore, the reliable morphological character to distinguish exactly the two species is only the single key character shown in Sato (2020) as follows: the heterogomph spinigers present among upper neurochaetae around chaetiger 5 in C. tokashikiensis, whereas those absent there in C. kumensis.

Need for conservation of small estuaries in the Ryukyu Islands. In the Ryukyu Islands, each of estuaries is limited in a narrow area of the lower reaches of small rivers or streams. The habitats of the two species of Composetia are extremely limited around the uppermost reaches of the small estuaries, and consequently most easily lost by human activities. In fact, the small estuaries have been seriously damaged, more recently, by the direct effect of anthropogenic coastal reclamations and concrete-revetment or seawall constructions, and also by the indirect effect of inland development such as dam constructions, which decrease the freshwater supply to the estuaries, sometimes resulting in estuary closing in the Ryukyu Islands (Ooshiro 2003; Diving Team Snack Snufkin 2015; Sato 2016). Some local populations of the two species of Composetia (particularly those in habitats of the stream type) may have been extirpated due to anthropogenic habitat loss, before we are aware of their existence. Therefore, C. kumensis [as Ceratonereis (Composetia) sp.] was designated as a threatened species (Data Deficient) by Japanese Association of Benthology (2012). Conservation and restoration of estuaries along the coasts of the Ryukyu Islands are strongly desirable to keep the unique fauna in- cluding the two species of Composetia.

\section{Acknowledgments}

We are grateful to Naoto Jimi (Hokkaido University), Kotaro Kan and Takeru Sakaguchi (Kagoshima University) for help in collecting materials, and to Hironori Komatsu (National Museum of Nature and Science) for identification of crabs. We also thank Bo Causer (Kagoshima University) for her English revising, and Tulio Fabio Villalobos-Guerrero (El Colegio de la Frontera Sur) and an anonymous reviewer for their helpful comments to the manuscript. The present study was partially supported by Special Budget of Ministry of Education, Culture, Sports, Science and Technology (Establishment of Research and Education Network on Biodiversity and Its Conservation in the Satsunan Islands), and by JSPS KAKENHI grant (no. 17H01913 and JP17K07538).

\section{References}

Angsupanich, S. and Rakkheaw, S. 1997. Seasonal variation of phytoplankton community in Thale Sap Songkhla, a lagoonal lake in southern Thailand. Netherlands Journal of Aquatic Ecology 30: 297-307.

Bakken, T. and Wilson, R. S. 2005. Phylogeny of nereidids (Polychaeta, Nereididae) with paragnaths. Zoologica Scripta 34: 507-547.

Diving Team Snack Snufkin (Ed.) 2015. Oura-wan no Ikimono-tachi [Life in Oura Bay]. Nanpou Shinsha, Kagoshima, 123 pp. [In Japanese]

Fukushima, M. and Ishida, A. 1966. [Aquaculture and life history of Perinereis brevicirris (Grube)]. The Aquiculture 14: 51-56. [In Japanese]

Hasegawa, T., Yamaguchi, T., Kojima, S., and Ohta, S. 1996. Phylogenetic analysis among three species of intertidal barnacles of the genus Tetraclita (Cirripedia; Balanomorpha) by nucleotide sequences of a mitochondrial gene. Benthos Research 51: 33-39. [In Japanese]

Huelsenbeck, J. P. and Ronquist, F. 2001. MRBAYES: Bayesian inference of phylogenetic trees. Bioinformatics 17: 754-755.

Japanese Association of Benthology (Ed.) 2012. Threatened Animals of Japanese Tidal flats: Red Data Book of Seashore Benthos. Tokai University Press, Hadano, 285 pp. [In Japanese]

Kikuchi, E. and Yasuda, K. 2006. Comparison of the life cycles of two sympatric estuarine polychaetes, Hediste diadroma and $H$. atoka (Polychaeta: Nereididae), in the Nanakita River estuary, northeastern Japan. Limnology 7: 103-115.

Kimura, M. 1980. A simple method for estimating evolutionary rate of base substitutions through comparative studies of nucleotide sequence. Journal of Molecular Evolution 16: 111-120.

Kojima, S., Segawa, R., Hashimoto, J., and Ohta, S. 1997. Molecular phylogeny of vestimentiferans collected around Japan, revealed by the nucleotide sequences of mitochondrial DNA. Marine Biology 127: 507-513.

Levin, L. A., Boesch, D. F., Covich, A., Dahm, C., Erséus, C., Ewel, K. C., Kneib, R. T., Moldenke, A., Palmer, M. A., Snelgrove, P., Strayer, D., and Weslawski, J. M. 2001. The function of marine critical transition zones and the importance of sediment biodiversity. Ecosystems 4: 430-451.

Maeda, K. and Tachihara, K. 2006. Fish fauna in the Teima Stream, Okinawa Island. Biological Magazine Okinawa 44: 7-25. [In Japanese] 
Obuchi, M. 2015. [Biodiversity in Oura Bay, Okinawa-jima island]. Science Journal Kagaku 85: 143-149. [In Japanese]

Ooshiro, M. 2003. [Effect of artificial constructions on the river organisms]. Pp. 65-72. In: Nishida, M., Shikatani, N., and Shokita, S. (Eds) The Flora and Fauna of Inland Waters in the Ryukyu Islands. Tokai University Press, Hadano. [In Japanese]

Ronquist, F. and Huelsenbeck, J. P. 2003. MrBayes 3: Bayesian phylogenetic inference under mixed models. Bioinformatics 19: 15721574.

Sato, M. 1999. Divergence of reproductive and developmental characteristics in Hediste (Polychaeta: Nereididae). Hydrobiologia 402: 129-143.

Sato, M. 2016. [Benthic animals in coral reefs and estuaries]. Pp. 247253. In: Study Group for Researches of Biodiversity in Kagoshima University (Ed.) Amami-gunto no Seibutsu Tayousei [Biodiversity in the Amami Islands]. Nanpo-shinsha, Kagoshima. [In Japanese]

Sato, M. 2017. Nereididae (Annelida) in Japan, with special reference to life-history differentiation among estuarine species. Pp. 477-512. In: Motokawa, M. and Kajihara, H. (Eds) Species Diversity of Animals in Japan. Springer Japan, Tokyo.

Sato, M. 2020. Two new species of Composetia (Annelida: Nereididae) from small estuaries in the Ryukyu Islands, southern Japan, with a list of all species currently belonging to Composetia. Species Diversity 25: 11-24.

Sato, M. and Nakashima, A. 2003. A review of Asian Hediste species complex (Nereididae, Polychaeta) with descriptions of two new species and a redescription of Hediste japonica (Izuka, 1908). Zoological Journal of the Linnean Society 137: 403-445.

Sato, M. and Sakaguchi, T. 2016. Nereidid polychaetes inhabiting the land-sea interface in the Amami Islands. Occasional Papers of Research Center for the Pacific Islands, Kagoshima University 57: 83-85. [In Japanese]

Sato, M. and Tsuchiya, M. 1991. Two patterns of early development in nereidid polychaetes keying out to Neanthes japonica (Izuka). Ophelia, Supplement 5: 371-382.

Schroeder, P. C. and Hermans, C. O. 1975. Annelida: Polychaeta. Pp. 1-213. In: Giese, A. C. and Pearse, J. S. (Eds) Reproduction of Marine Invertebrates. Vol. III. Academic Press, New York.

Shimayama, T., Himeno, H., Sasuga, J., Yokobori, S., Ueda, T., and Watanabe, K. 1990. The genetic code of a squid mitochondrial gene. Nucleic Acids Symposium Series 22: 77-78.

Stamatakis, A. 2006. RAxML-VI-HPC: maximum likelihood-based phylogenetic analyses with thousands of taxa and mixed models. Bioinformatics 22: 2688-2690.

Tamura, K., Peterson, D., Peterson, N., Stecher, G., Nei, M., and Kumar, S. 2011. MEGA5: molecular evolutionary genetics analysis using maximum likelihood, evolutionary distance, and maximum parsimony methods. Molecular Biology and Evolution 28: 2731-2739.

Thorson, G. 1950. Reproductive and larval ecology of marine bottom invertebrates. Biological Reviews 25: 1-45.

Uehara, A. and Nishijima, S. 2003. [Fishes in estuaries]. Pp. 488-489. In: Nishida, M., Shikatani, N. and Shokita, S. (Eds) The Flora and Fauna of Inland Waters in the Ryukyu Islands. Tokai University Press, Hadano. [In Japanese]

Yoshida, S. 1984. Studies on the biology and aquaculture of a common polychaete, Perinereis nuntia (Grube). Bulletin of the Osaka Prefectural Fisheries Experimental Station 6: 1-63. 